\title{
Private Rechtsetzung im Schatten des Gesetzes
}

\section{Ein Beitrag zur Bindungswirkung privaten Rechts am Beispiel des Deutschen Corporate Governance Kodex und der Deutschen Rechnungslegungs Standards}

Von Prof. Dr. Thomas M. J. Möllers* und Benjamin FekonjA**, Augsburg

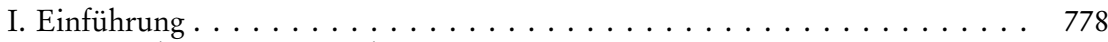

1. Ursachen privater Rechtsetzung . . . . . . . . . . . . . . . 778

2. IFRS, DRSC, DCGK als Beispiele privater Rechtsetzung . . . . . . . . . 780

3. Unklare Einordnung privater Rechtsetzung in das Rechtssystem . . . . . 781

II. Lehre von den sekundären Rechtsquellen . . . . . . . . . . . . . . . . 785

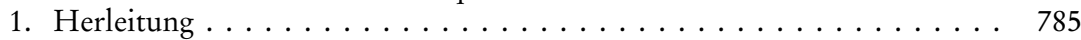

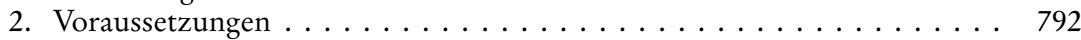

3. Rechtsfolgen ........................ 796

III. Deutsche Rechnungslegung Standards . . . . . . . . . . . . . . . . . . . 798

1. \342 HGB als hoheitlicher Rezeptionsakt . . . . . . . . . . . . . . . 798

2. Der Inhalt der Vermutungswirkung . . . . . . . . . . . . . . . . . . 799

3. Normsetzende Standards intra legem . . . . . . . . . . . . . . . . . . 800

4. Fehlende Vermutungswirkung bei normsetzenden Standards extra legem und contra legem . . . . . . . . . . . . . . . . . . . . 801

5. Widerlegung der Vermutungswirkung bei nicht überzeugenden Empfeh-

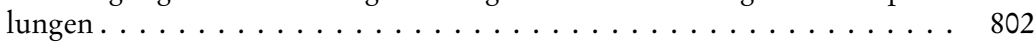

IV. Deutscher Corporate Governance Kodex . . . . . . . . . . . . . . . . . . . . . 803

1. \161 AktG als hoheitlicher Rezeptionsakt . . . . . . . . . . . . . 803

2. Heranziehung des klassischen Auslegungskanons zur Bestimmung der Bindungswirkung im Bereich von intra legem-Regeln des DCGK . . . . . 804

3. Fehlende Bindungswirkung des DCGK . . . . . . . . . . . . . . . . . 809

4. Zulässige Normkonkretisierung von gesetzlichen Generalklauseln durch private Regelsetzer. . . . . . . . . . . . . . . . . . . . . . . . . . . 811

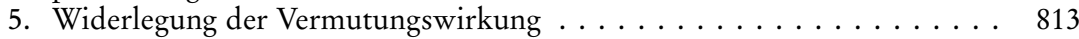

V. Zusammenfassung und Ausblick . . . . . . . . . . . . . . . . . . . 815

1. Zur Argumentationslast . . . . . . . . . . . . . . . . . . 815

2. Intra legem-Anbindung von Standards an das Gesetz . . . . . . . . . 815

* Dr. iur., Professor an der Universität Augsburg; Inhaber des Lehrstuhls für Bürgerliches Recht, Wirtschaftsrecht, Europarecht, Internationales Privatrecht und Rechtsvergleichung der Universität Augsburg, Geschäftsführender Direktor des Center for European Legal Studies (CELOS) sowie Inhaber eines Jean Monnet-Lehrstuhls ad personam.

** Ass. iur., Wirtschaftsjurist Univ. Bayreuth; Wissenschaftliche Hilfskraft am Lehrstuhl für Bürgerliches Recht, Wirtschaftsrecht, Europarecht, Internationales Privatrecht und Rechtsvergleichung der Universität Augsburg. 
Mit der klassischen Dichotomie von hard law und soft law, Rechtsquellen und Rechtserkenntnisquellen, lässt sich das Phänomen privater Normsetzung im Wirtschaftsrecht nicht mehr erfassen. Dagegen kann mit der Lehre von den sekundären Rechtsquellen die Rechtsverbindlichkeit von privater Regelsetzung angemessen umschrieben werden. Ähnlich wie im Bereich des Richterrechts trifft die Gerichte eine Befassungspflicht sowie eine subsidiäre Befolgungspflicht. Will der Adressat der Empfehlung nicht folgen, muss er dies gegenüber dem Gericht begründen. Weil der Gesetzgeber die wesentlichen Entscheidungen selbst treffen muss, kann der privaten Normsetzung eine solche mittelbar rechtliche Bindungswirkung nur zugestanden werden, wenn sie intra legem erfolgt, also eine Rückbindung an das demokratisch legitimierte Gesetz besteht. Im Einzelnen wird aufgezeigt, wann eine solche mittelbar rechtliche Bindungswirkung der Deutschen Rechnungslegungs Standards und den Empfeblungen des Deutschen Corporate Governance Kodex besteht und wann rechtlich nicht bindend eine Regelsetzung extra oder contra legem erfolgt.

The article establishes the claim that the traditional dichotomy of legal sources needs to be substituted by a trichotomy of hard law, soft law and a third kind of secondary law. The introduced doctrine of secondary sources of law adequately describes the binding effect of rules created by private entities such as the German Accounting Standards Committee's accounting principles (Deutsches Rechnungslegungs Standards Committee e. V.) and the rules of the German Corporate Governance Code (Deutscher Corporate Governance Kodex).

This so-called third kind of secondary law has three consequences: first of all, it needs to be recognized; secondly, it needs to be complied with; and thirdly, in case of non-compliance, extensive reasons must be given. This puts the third kind of secondary law in a position similar to that of persuasive authorities. Such position, however, can only be given to secondary law if it is sufficiently legitimized by the democratic lawmaker. Therefore, only intra legem rules can become legally binding, whereas extra or contra legem rules cannot be awarded this effect.

\section{Einfübrung}

\section{Ursachen privater Rechtsetzung}

Permanente Änderungen des regulatorischen Umfeldes stellen den parlamentarischen Gesetzgeber vor eine besondere Herausforderung. Technologische Innovationen und die Entwicklungsdynamik der Wirtschaft erfordern eine ungemeine Flexibilität des „klassischen“ staatlichen Rechts". Dem Gesetz als dem zentralen Steuerungsinstrument des demokratischen Rechtsstaates ${ }^{2}$ wird dabei seit geraumer Zeit eine nachlassende Steuerungskraft attestiert ${ }^{3}$ :

1 Lамв, Kooperative Gesetzeskonkretisierung, 1994, S. $35 \mathrm{ff}$.

2 Schuppert, Das Gesetz als zentrales Steuerungsinstrument des Rechtstaates, 1998; DERS., Governance und Rechtsetzung, 2011, S. 143.

3 Di Fabio, NZS 1998, 449 ff; OssenbüHL, in: Handbuch des Staatsrechts, 3. Aufl., 2007, $\$ 100$ Rdn. 71; BECKer, Kooperative und konsensuale Strukturen in der Normsetzung, 2005, S. 3 ff; a. A. ReImer, in: Hoffman-Riem/Schmidt-Assmann/Voßkuhle, Grundlagen des Verwaltungsrechts Bd. 1, 2. Aufl., 2012, $\$ 9$ Rdn. 84 ff. 
Das starre Korsett staatlicher Rechtsetzungsverfahren und der abstrakt-generelle Charakter staatlicher Handlungsinstrumente scheinen in Anbetracht einer dynamischen und globalen Wirtschaft seltsam antiquiert. Der Staat versucht dieses Defizit mittels einer ungebremsten Normenflut Herr zu werden ${ }^{4}$. Gleichzeitig kommt es zur oftmals zitierten „Flucht“ in die Generalklauseln und unbestimmten Rechtsbegriffe ${ }^{5}$. Auf Seiten der Normadressaten führt diese Entwicklung zu enormer Unsicherheit.

Bereits früh versuchte der Gesetzgeber dieser Entwicklung durch Kooperation mit den Normadressaten entgegenzutreten. Geschah dies auf dem Gebiet des Technikrechts anfangs vor allem wegen fehlender Information und fehlenden Sachverstandes staatlicher Stellen ${ }^{6}$, tritt heutzutage immer mehr ein weiteres Motiv in den Vordergrund: Die global agierenden Normadressaten sollen an der Gestaltung des nationalen regulatorischen Umfeldes partizipieren und damit gleichsam „ins Boot“ geholt werden”. Die Ausarbeitung von Regelwerken unter Einbindung von privaten Experten aus Wirtschaft und Wissenschaft wird daher seit geraumer Zeit mit den Begriffen der regulierten Selbstregulierung und der kooperativen Rechtsetzung verbunden ${ }^{8}$. Köndgen bezeichnete diese Form der staatlich initiierten privaten Rechtsetzung treffend als rule-making in the shadow of the law 9 . Private ${ }^{10}$ sollen nicht mehr nur Adressaten hoheitlicher Regulierung sein, sondern vielmehr selbst Verantwortung bei der rechtlichen Gestaltung ihres gesellschaftlichen Umfeldes übernehmen, indem sie Standards und Empfehlungen setzen können.

4 Isensee, ZRP 1985, 139 ff; Vogenauer, Die Auslegung von Gesetzen, 2000, S.174; kritisch zum Vorwurf der Normenflut hingegen, s. OssenbüHL, aaO (Fn. 3), $\$ 100$ Rdn. 77.

5 Hedemann, Flucht in die Generalklauseln, 1933; zur normstrukturellen Charakteristika im Wirtschaftsrecht, s. Hecker, Marktoptimierende Wirtschaftsaufsicht, 2007, S. $113 \mathrm{ff}$.

6 Marburger, Die Regeln der Technik im Recht, 1979, S. 2; Lamb, aaO (Fn. 1), S. 65.

7 Zur daraus resultierenden Verschränkung von Hierarchie und Verhandlung, s. BECKER, aaO (Fn. 3), S. 49 f.

8 S. dazu: Eifert, in: Hoffman-Riem/Schmidt-Assmann/Voßkuhle, Grundlagen des Verwaltungsrechts, Bd. 1, 2. Aufl., 2012, $\$ 19$ Rdn. 52 ff; Vosskuhle, VVDStRL 62 (2003), $266 \mathrm{ff}$.

9 Köndgen, AcP 206 (2006), 477, 496 ff; Die Formulierung wurde von ihm der amerikanischen Rechtssoziologie entnommen, MnOokin/Kornhauser, 88 Yale L.J. 950, 968 (1978/79).

10 Private sind all diejenigen natürlichen und juristischen Personen, die nicht der Staatsverwaltung angehören; Augsberg, Rechtsetzung zwischen Staat und Gesellschaft, 2003, S. 34. 


\section{IFRS, DRSC, DCGK als Beispiele privater Rechtsetzung}

Als Ausgangspunkt durchaus hilfreich ist die Formulierung Standards als das zu beschreiben, „was regelhaft daherkomme, aber nicht zwingendes Recht ist" ${ }^{11}$ oder Standards, die eine „gefühlte Nähe zum Recht“ aufweisen und sowohl bestimmte Handlungsanweisungen als auch -empfehlungen vorschreiben ${ }^{12}$. Regulative Standards weisen Rechtsnormcharakter auf und versuchen somit zu steuern. Sie kompensieren die Steuerungsschwäche des klassischen staatlichen Rechts, indem sie die tatbestandliche Offenheit des Rechts reduzieren (Norminterpretation und -konkretisierung). Gleichzeitig schließen sie Normierungslücken, die mangels Gesetzgebungskompetenz oder politischen Willens des Gesetzgebers entstanden sind (Normerzeugung) ${ }^{13}$. Beide Aufgaben erfolgen oftmals gleichzeitig durch ein und denselben Verhaltensstandard.

Im Wirtschaftsrecht fallen gleich mehrere private „Normsetzer“ ins Auge. Da sind zum einen die International Financial Reporting Standards (IFRS), die in Großbritannien durch das International Accounting Standards Board (IASB) festgelegt und dann im Rahmen des Komitologie-Verfahrens weitgehend in europäische Verordnungen umgesetzt werden ${ }^{14}$. Auf deutscher Seite zu nennen sind die Deutschen Rechnungslegung Standard des Deutschen Rechnungslegungs-Standards Committee e.V. (DRSC) ${ }^{15}$, die eine besondere rechtliche Bedeutung durch die Vermutungswirkung des $\$ 342 \mathrm{Abs}$. 2 HGB erlangt haben. Durch die comply-or-explain-Erklärung des $\$ 161 \mathrm{AktG}$ haben auch die Empfehlungen des Deutschen Corporate Governance Kodex (DCGK) der von der Bundesregierung eingesetzten Regierungskommission besondere Relevanz erhalten ${ }^{16}$.

11 Windbichler, in: Möllers, Geltung und Faktizität von Standards, 2009, S. 19.

12 Lepsius, in: Ch. Möllers/Voßkuhle/Walter, Internationales Verwaltungsrecht, 2007, S. 347 f. S. bereits: Möllers, Geltung und Faktizität von Standards, 2009, S. 143, 158.

13 So auch die Unterscheidung von SPINDLER/Hupka, in: Möllers, Geltung und Faktizität von Standards, 2009, S. 117, 124 f; Weber-Rey, ZGR 2010, 543, 555.

14 S. etwa Hirte/Mock, Kölner Komm. z. WpHG, 2007, \37n Rdn. 103, 105; Grund-

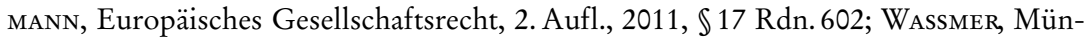
chener Komm. z. Bilanzrecht, 2012, Vorb. $\int \$ 331-335$ b HGB Rdn. 34 ff.

15 Deutsche Rechnungslegungs Standards (DRS) des Deutschen Rechnungslegungs Standards Committee e.V. (DRSC), Übersicht abrufbar unter: http://www.standardsetter.de/drsc/dsr/standards/index.php. Für die eigentliche Normsetzung zuständig ist innerhalb des DRSC der Deutsche Standardisierungsrat (DRS) als unselbständiges, aber unabhängiges Gremium ( $\$ 6$ Abs. 1 der Satzung des DRSC); s. BucK-HeEb/DiecKmann, Selbstregulierung im Privatrecht, 2010, S.129. Am 2.12.2011 wurde zwischen dem Bundesministerium der Justiz und dem DRSC ein neuer Standardisierungsvertrag geschlossen, abrufbar unter: http://www.standardsetter.de/drsc/docs/press_releases/ 2011/111202_SV_BMJ-DRSC.pdf.

16 Deutscher Corporate Governance Kodex in der Fassung v. 15. 5. 2012, abrufbar unter: http://www.corporate-governance-code.de/ger/kodex/index.html. 


\section{Unklare Einordnung privater Rechtsetzung in das Rechtssystem}

\section{a) Rechtliche Einordnung und Bindungswirkung}

Obwohl diese Normen nun schon zehn Jahre und länger existieren, herrscht immer noch Ungewissheit darüber, wie diese rechtsdogmatisch einzuordnen $\operatorname{sind}^{17}$. Eindeutig ist die dogmatische Einordnung bei den IFRS. Sie werden durch das Endorsement-Verfahren in europäische Verordnungen transformiert ${ }^{18}$. Als europäisches Sekundärrecht erlangen die „EU-IFRS“ Rechtsqualität und entfalten damit für das deutsche Recht Bindungswirkung ${ }^{19}$.

Große Unsicherheit herrscht hingegen bei der Einordnung der einzelnen privaten Standards, die nicht durch ein geregeltes Verfahren in verbindliches Recht transformiert werden. Symptomatisch dafür ist die nun seit einem Jahrzehnt in der rechtswissenschaftlichen Literatur geführte Diskussion zur Rechtsnatur des Deutschen Corporate Governance Kodex (DCGK). Weitgehende Einigkeit besteht darin, dass die Kodexbestimmungen keinen Handelsbrauch i.S.d. $\$ 346$ HGB darstellen und auch nicht zu Gewohnheitsrecht erstarken können ${ }^{20}$. Dafür fehlt es aufgrund der durch $\$ 161 \mathrm{AktG}$ eröffneten Abweichungsmöglichkeit an der erforderlichen Einheitlichkeit der Übung ${ }^{21}$. Zudem ist der Kodex gerade darauf angelegt, innerhalb relativ kurzer Zeit von der Kommission überarbeitet zu werden, so dass es auch an der für den Handelsbrauch erforderlichen Dauerhaftigkeit als Zeitmoment fehlt ${ }^{22}$. Mangels Rechtsgeltungswillens scheidet zudem die Einstufung als Gewohnheitsrecht aus ${ }^{23}$. Die sog. „opinio neccesitatis“ setzt voraus, dass die beteiligten Kreise davon überzeugt sind, durch Einhaltung einer Übung

17 LeYens, Großkomm. z. AktG, 4. Aufl., 2012, \$161 Rdn. 100.

18 Verordnung EG Nr. 1606/2002 des Europäischen Parlaments und des Rates v. 19. 7. 2002 betreffend die Anwendung internationaler Rechnungslegungsstandards, ABl. Nr. L 243 v. 11. 9.2002, S. 1 ff; Verordnung EG Nr. 1126/2008 der Kommission v. 3.11.2008 zur Übernahme bestimmter internationaler Rechnungslegungsstandards gemäß der Verordnung (EG) Nr. 1606/2002 des Europäischen Parlaments und des Rates, ABl. Nr. L 320 v. 29.11.2008, S. 1 ff. Zum Endorsement-Verfahren und den weiteren von der Kommission erlassenen Durchführungsverordnungen: Möllers, in: Schulze/Zuleeg/Kadelbach, Handbuch Europarecht, 2. Aufl., 2010, $\$ 18$ Rdn. 51 Fn. 131; HoHL, Private Standardsetzung im Gesellschafts- und Bilanzrecht, 2007, S. $140 \mathrm{ff}$.

19 Wassmer, aaO (Fn. 14), Vorb. $\$ \int 331-335$ b HGB Rdn. 34; Luttermann, WPg 2006, 778, 779; Schulze-Osterloh, Der Konzern 2004, 173.

20 Lutter, Kölner Komm. z. AktG, 3. Aufl., 2006, \$161 Rdn. 11; Kort, FS K. Schmidt, 2009, S. 945, 955 f; SpIndler, in: K. Schmidt/Lutter, AktG, 2. Aufl., 2010, $\$ 161$ Rdn. 8; Weber-Rey/Buckel, AG 2011, 845, 846; Mülbert/Wilhelm, ZHR 176 (2012), 286, 314.

21 Lutter, aaO (Fn. 20), \161 AktG Rdn. 11; Leyens, aaO (Fn. 17), \161 AktG Rdn. 105.

22 Semler, Münchener Komm. z. AktG, 2. Aufl., 2003, $\$ 161$ Rdn.33; Weber-Rey/ Buckel, AG 2011, 845, 846.

23 Spindler, aaO (Fn. 20), $\$ 161$ AktG Rdn. 8; Kort, FS K. Schmidt, S. 945, 955. 
bestehendes Recht zu befolgen ${ }^{24}$. Die durch den Gesetzgeber eingeräumte Möglichkeit zur freiwilligen Befolgung der Kodexempfehlungen spricht somit gegen einen solchen Rechtsgeltungswillen der kapitalmarktorientierten Unternehmen ${ }^{25}$.

Von der überwiegenden Literatur wird der DCGK als „soft law“ bezeichnet ${ }^{26}$. Die Kodexbestimmungen könnten zur Auslegung von gesetzlichen Bestim-

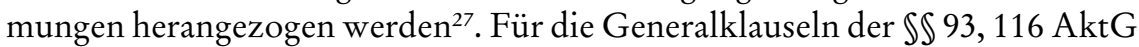
sollten die Kodexempfehlungen, unabhängig vom Inhalt der Entsprechenserklärung, als Standard guter Unternehmensführung dienen ${ }^{28}$. Würden die börsennotierten Unternehmen die Empfehlungen der Regierungskommission einhalten, hätte dies die Vermutung sorgfaltsgemäßen Handelns zur Folge ${ }^{29}$. Die Gegenansicht lehnt dies strikt ab und betont, dass die Regeln des DCGK rechtlich unverbindlich sind ${ }^{30}$. Die Möglichkeit, Hauptversammlungsbeschlüsse anzufechten ${ }^{31}$, vermenge "hard law“ und „soft law“32. Nach herr-

24 Kort, FS K. Schmidt, S. 945, 955.

25 Kort, FS K. Schmidt, S. 945, 955; Weber-Rey/Buckel, AG 2011, 845, 846.

26 Lutter, ZGR 2000, 1, 18; Ders., ZGR 2001, 224, 225; v. Werder, DB 2002, 801; E. VetTer, DNotZ 2003, 748, 754; Körner, NZG 2004, 1148, 1150; Kort, AG 2008, 137, 138; Linnerz, BB 2008, 581, 582; Semler, aaO (Fn. 22), \161 AktG Rdn. 28. Krit. zu diesem seinerseits interpretationsbedürftigen Begriff: Ulmer, ZHR 166 (2002), 150, 161; Ders. AcP 202 (2002), 143, 169; MüLbert, Arbeitspapiere des Instituts für deutsches und internationales Recht des Spar-, Giro- und Kreditwesens, Universität Mainz 2012, S. 27 f, abrufbar unter: http://www.jura.uni-mainz.de/muelbert/Dateien/Muelbert_Rechtsfragen_rund_um_den_DCGK.pdf.

27 Leyens, aaO (Fn. 17), \$161 AktG Rdn.102; Lutter, in: Ringleb/Cremer/Lutter/v. Werder, DCGK, 4. Aufl., 2010, Rdn.1623; Seibt, AG 2002, 249, 250 f; Doralt, in: Semler/v. Schenck, Arbeitshandbuch für Aufsichtsratsmitglieder, 2. Aufl., 2004, $\mathbb{} 13$ Rdn. 181.

28 LutTer, aaO (Fn. 20), $\mathbb{1} 161$ AktG Rdn. 81; Ders., in: Hommelhoff/Hopt/v. Werder, Handbuch Corporate Governance, 2. Aufl., 2009, S. 123, 133.

29 Die dogmatische Begründung für diese Vermutungswirkung ist dabei unterschiedlich: SEIBT, AG 2002, 249, 251 („Umkehrung der Beweislast“); Ulmer, ZHR 166 (2002), 150, 163; Fleischer, ZHR 168 (2004), 673, 707; Windidchler, aaO (Fn. 11), S. 19, 32 („safe harbour rules“); Hanfland, Haftungsrisiken im Zusammenhang mit $\$ 161 \mathrm{AktG}$ und dem DCGK, 2007, S. 93, 104 („prima facie-Beweis“); KoRT, FS K. Schmidt, S. 945, 959 („Indizfunktion unter Beibehaltung der Beweislast“); Weber-Rey/Buckel, AG 2011, 845, $848 \mathrm{ff}$ („mittelbare Haftungsrelevanz im Rahmen der business judgement rule").

30 So deutlich Hommelhoff/Schwaв, in: Hommelhoff/Hopt/v. Werder, Handbuch Corporate Governance, 2. Aufl., 2009, S. 71, 77; Habersack, Gutachten DJT 2012, E 45; Hoffmann-Becking, FS Hüffer, 2010, S. 337, 343; aber auch Lutter, aaO (Fn. 28), S. 123, 133: „der Kodex hat keine unmittelbare Wirkung“.

31 BGHZ 180, 9 - Kirch/Deutsche Bank; BGHZ 182, 272 - Springer; kritisch aber HofFMANN-BECKING, ZIP 2011, 1173, 1175; Kremer, ZIP 2011, 1177, 1179; WACLAwIK, ZIP 2011, 885, 891; TRÖGER, ZHR 175 (2011), 746, 772 ff.

32 Hoffmann-Becking, ZIP 2011, 1173, 1174 f. Ähnlich s. Pietrancosta, FS Hopt, 2010, 
schender Ansicht können auch die Rechnungslegung Standards des DRSC keine rechtliche Verbindlichkeit beanspruchen ${ }^{33}$. Da es sich um ein Privatrechtssubjekt handle, stünde dem privaten Rechnungslegungsgremium keine originäre Rechtsetzungsbefugnis zu.

\section{b) Verfassungsrechtliche Zweifel}

Schließlich werden auch verfassungsrechtliche Zweifel an der privaten Normsetzung vorgetragen. Die mit den Deutschen Rechnungslegungsvorschriften des DRSC verbundene Vermutungswirkung des $\ 342$ Abs. 2 HGB wird für verfassungswidrig erachtet ${ }^{34}$. Auch für den DCGK werden verfassungsrechtliche Bedenken vorgetragen ${ }^{35}$, wobei in jüngerer Zeit zwischen dem Kodex und der gesetzlichen Pflicht zur Abgabe einer Entsprechenserklärung nach $\$ 161 \mathrm{AktG}$ unterschieden wird ${ }^{36}$. Dabei wird die Regelsetzung des DCGK als verfassungskonform erachtet, da die Empfehlungen und Anregungen aufgrund ihrer rechtlichen Unverbindlichkeit nicht zu einem Grundrechtseingriff führen würden; im Ergebnis wird das Regelwerk der Regierungskommission als staatlich initiiertes, schlicht hoheitliches Informationshandeln der Bundesregierung verstanden ${ }^{37}$. Im Gegensatz dazu wird in der Literatur die comply-and-or-Regel des $\$ 161 \mathrm{AktG}$ als dynamische Verweisung qualifiziert und die Norm für verfassungswidrig erachtet, weil sie gegen die Wesentlichkeitstheorie verstoße ${ }^{38}$. Zudem stößt das Normsetzungsverfahren der Regie-

S. 1109, 1125: Verbindung von „soft law“ und Entsprechungserklärung als „hard law (soft law 2.0)“ und WeIss („Hybride Regulierungsinstrumente“), DERs., Hybride Regulierungsinstrumente, 2011, S. $100 \mathrm{ff}$.

33 Hommelhoff/Schwab, Großkomm. z. HGB, 5. Aufl., 2011, $\ 342$ Rdn. 89. S. dazu auch: KöndGEN, AcP 206 (2006), 477, 490.

34 Hommelhoff/Schwab, aaO (Fn. 33), \342 HGB Rdn. 12, 15 ff, 89 ff; Schuppert/ Bumke, in: Kleindiek/Oehler, Die Zukunft des deutschen Bilanzrechts, 2000, S. 71, 124; P. Kirchно, ZGR, 2000, 681, 681 f; a. A. Hohl, aaO (Fn. 18), S. 318.

35 Die Verfassungswidrigkeit bejahend: HüfFer, AktG, 10. Aufl., 2012, 1161 Rdn.4; Spindler, aaO (Fn. 20), $\$ 161$ AktG Rdn. 11; SeIdel, ZIP 2004, 285 ff; a. A. z. B. BACHMANN, WM 2002, 2137, 2142; HeINTZEN, ZIP 2004, 1933, 1936; KoRT, 5 ECFR 379, 415 (2008); Lutter, aaO (Fn. 20), \$161 AktG Rdn. 12; Kirschbaum/Wittmann, JuS 2005, 1062, 1064; HoHL, aaO (Fn. 18), S. $111 \mathrm{f}$.

36 Hoffmann-Becking, FS Hüffer, S. 336 ff; Wernsmann/Gatzka, NZG 2011, 1001 ff; MÜlberT/Wilhelm, ZHR 176 (2012), 286, $312 \mathrm{ff}$.

37 Wernsmann/Gatzka, NZG 2011, 1001, 1004 ff; Mülbert, aaO (Fn. 26), S. 26, 27 f; DERs./Wilhelm, ZHR 176 (2012), 286, 314 ff; vorher schon Hoffmann-Becking, FS Hüffer, S. 336, 340 f; HeInTZEN, ZIP 2004, 1933, 1934; Zur Rechtsprechung des BVerfG s. BVerfGE 105, $252 \mathrm{ff}$ - Glykol; BVerfGE 105, $279 \mathrm{ff}$ - Osho.

38 Ausdrücklich Wernsmann/Gatzka, NZG 2011, 1001, 1007; Hoffmann-Becking, ZIP 2011, 1173, 1174; Mülbert, aaO (Fn. 26), S. 31. a. A. aber etwa Habersack, Gutachten DJT 2012, E 54; Vogel, Die Haftung von Gesellschaften, Vorständen und Auf- 
rungskommission auf Kritik. Im Gegensatz zu den Rechnungslegungsstandards fehle es an gesetzgeberischen Vorgaben zur Besetzung und zu Verfahren der Kommission ${ }^{39}$. Die Regierungskommission hat mit dem jüngst durchgeführten Konsultationsverfahren einen Schritt in die richtige Richtung gemacht, um diese Kritik zu entkräften ${ }^{40}$. Dieses erfolgte jedoch auf freiwilliger Basis. Will der Gesetzgeber dem Vorwurf der fehlenden demokratischen Legitimation begegnen, wird er nicht umhin kommen, das Konsultationsverfahren der Regierungskommission zumindest gesetzlich verpflichtend vorzuschreiben und Kriterien für die Besetzung des Gremiums und die Teilnehmer am Konsultationsverfahren zu normieren, so wie er dies für das private Rechnungslegungsgremium bereits getan hat ${ }^{41}$.

Im Ergebnis wird den Rechnungslegungsvorschriften oder den Regeln des DCGK zwar eine hohe faktische Wirksamkeit attestiert; die rechtliche Bindungswirkung bleibt jedoch unklar. Im Folgenden ist zu zeigen, dass die Zweispurigkeit von verbindlichen, durch den Staat gesetzten Rechtsnormen und unverbindlichem soft law überholt ist und von einer dritten Kategorie von Recht im Rahmen der Rechtsetzung Privater gesprochen werden kann, den sekundären Rechtsquellen. Diese zeichnen sich durch eine mittelbare Bindungswirkung aus, nämlich eine Befassungs- und Befolgungspflicht aufgrund der Vermutungswirkung. Mit diesen Rechtswirkungen lässt sich der Umfang der Rechtsverbindlichkeit privater Rechtsquellen genauer beschreiben als mit der bisher bekannten Dichotomie von „hard law“ versus „soft law“. Nach der rechtsdogmatischen Beschreibung dieser sekundären Rechtsquellen (II.) werden diese Überlegungen dann anhand der privaten Regelwerke der DRSC (III.) und der Regierungskommission Corporate Governance (IV.) an verschiedenen Fällen überprüft.

sichtsräten im Zusammenhang mit der Entsprechenserklärung zum DCGK gem. \161 AktG, 2011, S. 55 ff; Hanfland, aaO (Fn.29), S. 123 ff. Zur Wesentlichkeitstheorie sogleich II.2.b).

39 Spindler, aaO (Fn. 20), $\$ 161$ AktG Rdn.11; Hommelhoff/Schwab, aaO (Fn. 30), S. $83 \mathrm{f}$.

40 S. Schreiben der Regierungskommission DCGK v. 1.2.2012, abrufbar unter: http://www.corporate-governance-code.de/ger/download/aenderungen_2012/Vorspann_Klaus_Peter_Mueller.pdf.

41 Gem. $\$ 342$ Abs. 1 S. 2 HGB verlangt der Gesetzgeber, „dass die Empfehlungen und Interpretationen [des privaten Rechnungslegungsgremiums] unabhängig und ausschließlich von Rechnungslegern in einem Verfahren entwickelt und beschlossen werden, das die fachlich interessierte Öffentlichkeit einbezieht.“ Kritisch auch SpINDLER, aaO (Fn. 20), $\$ 161$ AktG Rdn. 11. Für ein obligatorisches Konsultationsverfahren auch: Habersack, Gutachten DJT 2012, E 54. 


\section{Lehre von den sekundären Rechtsquellen}

\section{Herleitung}

\section{a) Zum Begriff der Rechtsquelle}

Klassischerweise unterscheidet man zwischen Rechtsquelle und Rechtserkenntnisquelle: Als Rechtsquelle wird nur anerkannt, was für den Rechtsanwender einen verbindlichen Rechtssatz darstellt ${ }^{42}$. Im Unterschied dazu werden rechtlich nicht bindende Einflussfaktoren nur als unverbindliche Rechtserkenntnisquelle bezeichnet. Für Richterrecht ${ }^{43}$, Verwaltungsvorschriften $^{44}$ oder private Rechtsetzung ${ }^{45}$ wird regelmäßig nur von einer faktischen Bindungswirkung gesprochen. Erklärbar ist diese Trennung, weil bisher zwischen Rechtsetzung und Rechtsanwendung unterschieden wurde ${ }^{46}$.

\section{b) Die Bindungswirkung der Rechtsprechung}

Es gehört heutzutage zum gesicherten Bestand der Rechtstheorie, dass außer den primären Rechtsquellen auch das Richterrecht mehr als nur eine faktische Bindung erzeugt. Vor allem Kriele, Bydlinski und Alexy haben Präjudizien der Gerichte als sekundäre Rechtsquellen bezeichnet ${ }^{47}$. Danach müssen sich Gerichte mit den früheren einschlägigen Urteilen befassen ${ }^{48}$. Sodann wurde eine subsidiäre Befolgungspflicht der Gerichte festgestellt. Wenn mehrere Lösun-

42 RÜthers/Fischer/Birk, Rechtstheorie, 6. Aufl., 2012, Rdn. 217 f. Für einen weiteren soziologischen Rechtsquellenbegriff s. etwa Vesting, Rechtstheorie, 2007, Rdn. 185.

43 Enneccerus/Nipperdey, Allgemeiner Teil des Bürgerlichen Rechts, 15. Aufl., 1959, S. 206; Larenz, Methodenlehre der Rechtswissenschaft, 6. Aufl., 1991, S. 432; Picker, JZ 1988, 62, 72 f; KÖHLER, JR 1984, 45, 48.

44 BVerwG v. 6.11.1986, BVerwGE 75, 109, 115, 117 f; BVerwG v. 25. 11. 1993, BVerwGE 94, 335, 340; Maurer, Allgemeines Verwaltungsrecht, 18. Aufl., 2011, $\$ 24$ Rdn. 3 u. 20. Für die Verlautbarungen der BaFin etwa Köndgen, ZBB 1996, 361; Möllers/Ganten, ZGR 1998, 773, 801; Assmann, in: Assmann/Schneider, WpHG, 6. Aufl., 2012, Einl. Rdn. 67 ff; SCHÄDLE, Exekutive Normsetzung in der Finanzmarktaufsicht, 2007, S. 89.

45 Wagner, Münchener Komm. z. BGB, 5. Aufl., 2009, \823 Rdn. 280 ff.

46 Von Bogdandy, Gubernative Rechtssetzung, 2000, S.156; S. bereits Möllers, FS Buchner, 2009, S.649, 654; DERs., aaO (Fn. 12), S. 143, 150; RufFerT, in: HoffmanRiem/Schmidt-Assmann/Voßkuhle, Grundlagen des Verwaltungsrechts Bd.1, 2. Aufl., 2012, $\$ 17$ Rdn. 1.

47 Kriele, Theorie der Rechtsgewinnung, entwickelt am Problem der Verfassungsinterpretation, 2. Aufl., 1976, S. 243 ff; ByDlinski, Juristische Methodenlehre und Rechtsbegriff, 2. Aufl., 1991, S. 506 ff, und Alexy, Theorie der juristischen Argumentation, 4. Aufl., 2001, S. 339, S. bereits Möllers, FS Buchner, S. 649, 655; DERs., aaO (Fn. 12), S. $143,152$.

48 Vogel, Juristische Methodik, 1998, S. 86. 
gen möglich und annähernd gleichmäßig vertretbar sind, sind höchstrichterliche Entscheidungen in der Weise verbindlich, als sich die Argumentationslast ändert: Wer von früheren Urteilen abweichen will, muss dies besonders begründen ${ }^{49}$. Inzwischen geht auch die überwiegende Meinung von einer solchen subsidiären Befolgungspflicht oder Präjudizienvermutung aus ${ }^{50}$. Ein solches Ergebnis entspricht auch der Praxis des EuGH. Zwar binden dessen Präjudizien nicht im Sinne einer „stare decicis-rule“51, der Gerichtshof zitiert jedoch als Einstieg zu seiner Urteilsbegründung regelmäßig zunächst die zu der streitigen Rechtsfrage bereits bestehende Rechtsprechung und entwickelt ausgehend davon seine Argumentationslinie ${ }^{52}$. Bydlinski spricht deshalb von ergänzenden Rechtsquellen ${ }^{53}$.

\section{c) Gesetzlich angeordnete Vermutungstatbestände und europäische Empfeblungen}

Die bisherige Zweiteilung der Rechtsquellenlehre ist mithin um eine dritte Kategorie, die sekundären Rechtsquellen, zu erweitern, so dass künftig von einer Trichotomie zu sprechen ist ${ }^{54}$. Zu diesen sekundären Rechtsquellen können neben der bereits angesprochenen höchstrichterlichen Rechtsprechung Verwaltungsvorschriften, aber auch private Normsetzung gehören ${ }^{55}$. Dass es eine solche Trichotomie gibt, verdeutlichen drei Fälle, in denen der Gesetz-

49 Kriele, aaO (Fn. 47), S. 243 ff; Bydlinski, aaO (Fn. 47), S. 506 ff; Alexy, aaO (Fn. 47), S. 339 .

50 Fikentscher, Die Bedeutung der Präjudizien im heutigen deutschen Privatrecht, in: Blaurock, Die Bedeutung von Präjudizien im französischen und deutschen Recht, 1989, S. 11, 19; RAIsch, Juristische Methoden, 1995, S. 192; RüThers/Fischer/BIRK, aaO (Fn. 42), Rdn. 239 ff; Langenbucher, Die Entwicklung und Auslegung von Richterrecht, 1996, S. 105 ff; Möllers, Die Rolle des Rechts im Rahmen der europäischen Integration, 1999, S.70; LAREnz/CANARIs, Methodenlehre der Rechtswissenschaft, 3. Aufl., 1995, S. $256 \mathrm{f}$.

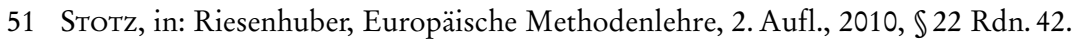

52 Zur Rechtsprechung des EuGH unter dem Gesichtspunkt der Vorhersehbarkeit und Kohärenz: Sтотz, аaO (Fn.51), $\$ 22$ Rdn.41; Grok, Die Auslegungsbefugnis des EuGH im Vorabentscheidungsverfahren, 2005, S. $180 \mathrm{ff}$.

53 So Bydlinski, JZ 1985, 149, 151 für das Richterrecht.

54 Im Folgenden ist statt von schwächerer oder ergänzender besser von sekundärer Rechtsquelle $\mathrm{zu}$ sprechen, um zu verdeutlichen, dass sich die private Norm auf das Gesetz oder zumindest staatliche Gewalt bezieht, s. Möllers, FS Buchner, S. 649, 654.

55 Möllers, FS Buchner, S. 649, 654 ff; DERs., aaO (Fn. 12), S. 143 ff; ihm folgend SPINDLER/Hupka, aaO (Fn. 13), 2008, S. 117, 118; Arndt, Sinn und Unsinn von Soft Law, 2010, S. 117 ff; Rötting/LAng, EuZW 2012, 8, 10 f; Blaurock, JZ 2012, 226 ff (Fn. 2); ähnlich: Siegel, NVwZ 2008, 620; Michel, DÖV 2011, 728, 732 („sonstige Rechtswirkungen"). 
geber oder die Rechtsprechung ausdrücklich eine Vermutungswirkung anordnen.

Bekanntestes Beispiel auf nationaler Ebene ist die im Bereich der Konzernrechnungslegung gesetzlich angeordnete Vermutungswirkung ( $\$ 342$ Abs. 2 HGB) für die Rechnungslegung Standards des DRSC, auf die im Folgenden intensiver eingegangen wird ${ }^{56}$.

Ein zweites Beispiel bildet die Empfehlung nach europäischem Recht, die auch im Europäischen Gesellschaftsrecht durchaus verbreitet is ${ }^{57}$. Nach Art. 288 Abs. 5 AEUV sind Empfehlungen von Unionsorganen zwar „nicht verbindlich. " ${ }^{58}$ Ungeachtet dieses Wortlautes verlangt der EuGH aber von den Mitgliedstaaten, die Empfehlungen der Kommission zur Auslegung innerstaatlicher, Gemeinschaftsrecht durchführender Rechtsvorschriften oder zur Ergänzung verbindlicher gemeinschaftlicher Vorschriften zu berücksichtigen $^{59}$. Als Adressaten hätten die Mitgliedstaaten die Empfehlung ernsthaft zu prüfen und sich nach ihnen zu richten oder sie aber mit ausreichender Erklärung zurückzuweisen ${ }^{60}$.

Ein drittes Beispiel bilden in jüngster Zeit Empfehlungen, die der europäische Gesetzgeber im Verhältnis der europäischen Finanzaufsichtsbehörden zu den nationalen Behörden gesetzlich normiert hat. Für die Empfehlung der Finanzaufsichtsbehörden EBA, ESMA und EIOPA ${ }^{61}$ hat der europäische Gesetz-

56 Ähnliche Regelungen finden sich in $₫ 4$ Abs. 1 S. 2 GPSG; $₫ 16$ Abs. 1 S. 2 TPG.

57 S. etwa Empfehlung der Kommission zur externen Qualitätssicherung bei Abschlussprüfern und Prüfungsgesellschaften, die Unternehmen von öffentlichem Interesse prüfen vom 7.5.2008, 2008/362/EG, ABl. Nr. L 120, 20; Empfehlung der Kommission zur Beschränkung der zivilrechtlichen Haftung von Abschlussprüfern und Prüfungsgesellschaften v. 5.6.2008, 2008/473/EG, ABl. Nr. L 162, 139; Empfehlung der EUKommission zu den Aufgaben von nicht geschäftsführenden Direktoren/Aufsichtsratsmitgliedern börsennotierten Gesellschaften sowie zu den Ausschüssen des Verwaltungs-/Aufsichtsrats v. 15. 2. 2005, 2005/162/EG, ABl. Nr. L 52, 51.

58 Vielmehr sollen sie lediglich zur „weichen“, influenzierenden Steuerung eingesetzt werden, Ruffert, in: Calliess/Ruffert, EUV AEUV, 4. Aufl., 2011, Art. 288 Rdn. 95; DERs., aaO (Fn. 46), \$17 Rdn. 37; FleIsCHER, ZGR 2012, 160, 177.

59 EuGH v. 13.12.1989, Rs. C-322/88, Slg. 1989, 4407, Rdn. 18 - Grimaldi/Fonds des maladies professionnelles.

60 EuGH v. 21.1.1993, Rs. C-188/91, Slg. 1993, I-363 Rdn. 18 - Shell: „Wenn die Empfehlungen des Gemischten Ausschusses auch keine Rechte für den einzelnen begründen können, auf die dieser sich vor den nationalen Gerichten berufen könnte, so sind die nationalen Gerichte dennoch verpflichtet, sie bei der Entscheidung der bei ihnen anhängigen Rechtsstreitigkeiten zu berücksichtigen, zumal wenn sie, wie beim Sachverhalt des Ausgangsverfahrens, für die Auslegung der Bestimmungen des Übereinkommens von Nutzen sind.“ MöLlers, ZFR-Spezial, Aktuelles Finanzmarktrecht, 2010, 1, 15.

61 European Banking Authority (EBA); European Securities and Markets Authority (ESMA); European Insurance and Occupational Pensions Authority (EIOPA). 
geber jeweils den gerade beschriebenen Mechanismus vorgesehen: Grundsätzlich hat die nationale Behörde der Aufsichtsbehörde zu bestätigen, dass sie der Empfehlung nachkommt. Will sie von der Empfehlung abweichen, hat sie der europäischen Finanzaufsichtsbehörde die Gründe mitzuteilen ${ }^{62}$. In all diesen Fällen geht der Gesetzgeber davon aus, dass der Adressat dem angeordneten Imperativ Folge leistet. Will der Adressat von der gesetzlich angeordneten Pflicht abweichen, muss er dies begründen ${ }^{63}$.

\section{d) Emittentenleitfaden, Rundschreiben und sonstige Verlautbarungen der BaFin}

\section{aa) Zum Streitstand}

Dogmatisch weitgehend ungeklärt ist auch die rechtliche Einordnung des nunmehr in zweiter Fassung veröffentlichten Emittentenleitfadens der BaFin $^{64}$. Von Rechtsprechung ${ }^{65}$ und herrschender Lehre ${ }^{66}$ werden der Emittentenleitfaden und die sonstigen Verlautbarungen der BaFin als norminterpretierende Verwaltungsvorschriften und damit als reine Innenrechtsakte der Behörde eingestuft, obwohl die enorme faktische Bindungswirkung dieser Regelwerke für die Marktteilnehmer allgemein anerkannt ist ${ }^{67}$. Schon seit

62 S. etwa für die ESMA: Art. 16 Abs. 3 Ua. 2 der Verordnung Nr. 1093/2010 des Europäischen Parlaments und des Rates vom 24.11.2010 zur Errichtung einer Europäischen Aufsichtsbehörde (Europäische Bankenaufsichtsbehörde), zur Änderung des Beschlusses Nr. 716/2009/EG und zur Aufhebung des Beschlusses 2009/78/EG der Kommission (ESMA-VO), ABl. Nr. L 331, 12. S. dazu auch: Möllers, NZG 2010, 285, 289; MöLLERs/Harrer/Krüger, WM 2011, 1537, 1543; Rötting/LANG, EuZW 2012, 8, 10 f.

63 Zum Vorschlag einer RatingVO und zur ESMA-VO s. bereits Möllers, 10 EBOR 2010, 379, 390 ff; DERs., NZG 2010, 285, $287 \mathrm{f}$.

64 Emittentenleitfaden der BaFin i.d.F. v. 27.4.2009, abrufbar unter: bttp://www.bafin.de/SharedDocs/Downloads/DE/Leitfaden/WA/dl_090520_emittentenleitfaden_ 2009.pdf?_blob=publicationFile.

65 BGH, BB 2012, 530, 534 - IKB; BGH, WM 2008, 641, 644 - Daimler Chrysler AG/ Schrempp; AG Frankfurt a. M. v. 15. 8. 2008, 934 OWi 7411J 233764/07 - BeckRS 2008, 19726; VGH Kassel, WM 2007, 392, 393; BGH, NJW 2006, 1429, 1431; OLG Düsseldorf, NJW-RR 2004, 409f; BGH, NJW 2002, 62, 63.

66 Für den Emittentenleitfaden: Claussen/Florian, AG 2005, 745, 747; Merkner/SustMANN, NZG 2005, 729, 730; Fleischer, NZG 2007, 401, 404; Nikoleyczik, GWR 2009, 264; weitergehend: BEDKOwsKI, BB 2009, 394, 399 („persuasive authority“); Für die sonstigen Verlautbarungen: Möllers/Ganten, ZGR 1998, 773, 806 f; BürkLe, VersR 2009, 866, 868; Weber-Rey, ZGR 2010, 543, 556; Braun, in: Boos/Fischer/Schulte-Mattler, KWG, 4. Aufl., 2012, $\$ 25$ a Rdn. 47. Allgemein zu norminterpretierenden Verwaltungsvorschriften: RufFerT, aaO (Fn. 46), $\$ 17$ Rdn. 74.

67 Bedkowski, BB 2009, 394; Burg/Marx, AG 2009, 487 f; Weber-Rey/Baltzer, in: Hopt/Wohlmannstetter, Handbuch Corporate Governance von Banken, 2011, S. 457 f; Habersack, Gutachten DJT 2012, E 54. 
geraumer Zeit wird im Verwaltungsrecht diese Unterscheidung zwischen Innen- und Außenrecht kritisiert, da sie nicht in der Lage sei, die enorme Steuerungsleistung von Verwaltungsvorschriften in angemessener Weise zu erfas$\operatorname{sen}^{68}$. Wabl bezeichnete diese Diskrepanz zwischen Rechtsdogmatik und Rechtstheorie treffend als „Dogmatik des Als-Ob.“69 Die herrschende Meinung ließe es zu, dass die Praxis die Verwaltungsvorschriften so behandeln würde, als ob sie eine dritte Kategorie des Rechts darstellen würden, versage ihnen jedoch gerade diese Qualität in der Dogmatik ${ }^{70}$. Dementsprechend konsequent möchte eine sich im Vordringen befindliche Meinung Verwaltungsvorschriften eine abgeschwächte rechtliche Bindungswirkung, ähnlich den Präjudizien, zusprechen ${ }^{71}$.

\section{bb) Entwicklungslinien der Rechtsprechung}

(1) Noch im Jahre 2008 ignorierte der 2. Senat des BGH bei der Frage, ob die Daimler Chrysler AG das Anlegerpublikum rechtzeitig von dem beabsichtigten Rücktritt des damaligen Vorstandsvorsitzenden Schrempp informiert hatte, die Auffassung der BaFin vollständig ${ }^{72}$. Er begnügte sich mit einem Hinweis auf dessen vermeintlich unverbindliche Rechtsnatur ${ }^{73}$ und überging damit die dezidierte Meinung der BaFin, die die Ad-hoc-Publizitätspflicht in mehrstufigen Entscheidungsprozessen nicht erst bei endgültiger Zustimmung durch den Aufsichtsrat entstehen $\operatorname{sah}^{74}$.

Umso erfreulicher ist es zu beobachten, dass sich der Bundesgerichtshof und einige Oberlandesgerichte dieser Meinung anzunähern scheinen und sich mittlerweile intensiv mit dem Emittentenleitfaden der BaFin auseinandersetzen.

Der 20. Senat des OLG Stuttgart, an den die Rechtssache Daimler Chrysler zurückverwiesen wurde, maß dem Emittentenleitfaden der BaFin hingegen

68 Ruffert, aaO (Fn. 46), $\$ 17$ Rdn. 67 f; Schuppert, aaO (Fn. 2), S. 189 ff; Schmidt-AssMANn, Das allgemeine Verwaltungsrecht als Ordnungsidee, 2. Aufl., 2006, S.328; v. Bogdandy, aaO (Fn. 46), S. $455 \mathrm{f}$.

69 Wahl, FG 50 Jahre BVerwG, 2003, S. 571, $582 \mathrm{ff}$.

70 Wahl, FG 50 Jahre BVerwG, 2003, S. 571, 585; so auch Schuppert, aaO (Fn. 2), S. 187 f.

71 Rogmann, Die Bindungswirkung von Verwaltungsvorschriften, 1998, S. 217, $221 \mathrm{ff}$; OssenbüHL, DVBl. 1999, 1, 5; v. Bogdandy, aaO (Fn. 46), S. 449 ff, 466 ff; Leisner, JZ 2002, 219; Sauerland, Die Verwaltungsvorschriften im System der Rechtsquellen, 2004, S. 274 ff, 352 ff; Schmidt-Assmann, aaO (Fn. 68), S. 328 f; Möllers, FS Buchner, S. $649,660 f$.

72 BGH, WM 2008, 641 - Daimler Chrysler AG/Schrempp.

73 BGH, WM 2008, 641, 644 - Daimler Chrysler AG/Schrempp.

74 Emittentenleitfaden der BaFin i.d.F. v. 15.7.2005, S. 44, 46 u. 50, abrufbar unter: http://www.bafin.de/SharedDocs/Downloads/DE/Leitfaden/WA/dl_emittentenleitfaden_2005.pdf;jsessionid,,CCD9362049B700A3BD48FB5BD1A57A49.1_cid226?_ blob,publicationFile9. Kritisch bereits: MöLlers, NZG 2008, 330, 331. 
eine deutlich höhere Bedeutung bei ${ }^{75}$. Der Senat kam zunächst zu dem Ergebnis, dass, entgegen der Auffassung der BaFin, ${ }^{76}$ die Frage, ob es sich um eine Insiderinformation i.S.d. $\$ 13$ Abs. 1 Satz 1 WpHG handele, nicht bei jeder einzelnen (Zwischen-) Stufe isoliert zu prüfen sei. Vielmehr könne $\$ 13$ Abs. 1 Satz 3 WpHG entnommen werden, dass es bei einem solchen gestreckten Geschehensablauf auf die hinreichende Wahrscheinlichkeit des Eintritts des angestrebten (End-) Ereignisses ankäme ${ }^{77}$. Die Zustimmung des Aufsichtsrats zum einvernehmlichen Ausscheiden des Vorstandsvorsitzenden Schrempp sei jedoch erst unmittelbar am Vorabend der Aufsichtsratssitzung durch den Beschluss des Präsidialausschusses „hinreichend wahrscheinlich“" gewesen ${ }^{78}$.

Für den Zeitraum zwischen der Vorabstimmung im Präsidialausschuss und der eigentlichen Beschlussfassung am Folgetag hätten hingegen nach Auffassung des Senats die Voraussetzungen für eine Befreiung nach $\$ 15$ Abs. 3 Satz 1 WpHG vorgelegen. Bei dieser Frage schloss sich das OLG Stuttgart der Auffassung der BaFin an und wies darauf hin, dass „auch die zuständige Verwaltungsbehörde“ davon ausgehe, „dass die gesetzlichen Aufgaben zur Überwachung des Vorstands regelmäßig eine Befreiung rechtfertigen“ würden ${ }^{79}$.

(2) Noch deutlicher wird die gesteigerte Bedeutung des Emittentenleitfadens in der höchstrichterlichen Rechtsprechung im Fall IKB ${ }^{80}$. Im Mittelpunkt stand die Haftung der IKB für eine Pressemitteilung ihres Vorstandsvorsitzenden im Zusammenhang mit ihrem Engagement in US-amerikanischen Subprime-Pa-

75 OLG Stuttgart, NZG 2009, $624 \mathrm{ff}$.

76 Emittentenleitfaden der BaFin i. d. F. v. 15.7.2005, S. 19. So auch: OLG Frankfurt a. M., NZG 2009, 391 ff; Möllers, WM 2005, 1393, 1394; Möllers/Bornemann, WuB I G 6 \13 WpHG 1.09; Simon, Der Konzern 2005, 13, 15; Pawlik, Kölner Komm. z. WpHG, 2007, \13 Rdn. 16; Mennicke/Jakovou, in: Fuchs, WpHG, 2009, \13 Rdn. 74.

77 OLG Stuttgart, NZG 2009, 624, 626; So auch: Assmann, aaO (Fn. 44), \13 WpHG Rdn. 28; Schwark/Kruse, in: Schwark/Zimmer, WpHG, 4. Aufl., 2010, \13 Rdn. 10 a.

78 OLG Stuttgart, NZG 2009, 624, 626. Der EuGH hat inzwischen entschieden, s. EuGH, Urt. v. 28. 6. 2012, Rs. C-19/11, Rdn. 38 ff - Geltl/Daimler AG, NZG 2012, 784 ff; hierzu Möllers/Seidenschwann, NJW 2012, 2762 ff. In seinem Schlussantrag in der Rs. Geltl/Daimler AG verweist GA Mengozzi ebenfalls ausdrücklich auf die vermeintlich unverbindlichen Leitlinien des mittlerweile aufgehobenen Europäischen Wertpapierauschusses (CESR): „Der Ausschuss der Europäischen Wertpapierregulierungsbehörden (...) stellte im Übrigen in seinen Leitlinien zur Anwendung der Richtlinie 2003/6 vom Juli 2007(9) in Nr. 1.6 fest, dass, wenn die Information einen Vorgang betrifft, der sich über mehrere Stufen erstreckt, jede einzelne Stufe des Vorgangs ebenso wie der Gesamtvorgang selbst eine präzise Information sein kann“, Schlussantrag GA Mengozzi v. 21. 3. 2012, Rs. C-19/11, Rdn. 55 - Geltl/DaimlerAG, BeckRS 2012, 80624.

79 OLG Stuttgart, NZG 2009, 624, 631 unter ausdrücklichem Verweis auf den Emittentenleitfaden der BaFin i.d. F. v. 15.7.2005, S. 55 und Möllers, WM 2005, 1393, 1398, 1400.

80 BGH, NJW 2012, 1800 ff - IKB; OLG Düsseldorf, OLG Düsseldorf v. 7. 4. 2011, I-6 U 7/10, 6 U 7/10 - juris; s. dazu auch: Hellgardt, DB 2012, 450 ff; v. BERnUtH/WaGNER/ Kremer, WM 2012, 831 ff; Seibt, EWiR 2012, 159 ff; Spindler, NZG 2012, 575 ff. 
pieren. Zu einem Zeitpunkt, in dem sich bereits abzeichnete, dass die IKB einem erhöhten Ausfallrisiko ausgesetzt war, veröffentlichte der IKB-Vorstandsvorsitzende Ortseifen eine Pressemitteilung, in der er "praktisch keine Auswirkungen " der Hypothekenkrise für die IKB prognostizierte. Eine Woche später war die IKB aufgrund der Sperrung von Kreditlinien gezwungen, unter einem von der BaFin initiierten sog. Rettungsschirm Schutz zu suchen.

Das OLG Düsseldorf sah in der Höhe des Subprime-Anteils keine konkrete veröffentlichungspflichtige Insiderinformation i.S.d. $\$ 13$ Abs. 1 Satz 1 $\mathrm{WpHG}^{81}$. Der Senat verwies darauf, dass auch die BaFin in ihrem Emittentenleitfaden „ein erhebliches Preisbeeinflussungspotential nur für konkrete und massive Ereignisse“, wie z. B. bei „wesentlichen Änderungen der Ergebnisse der Jahresabschlüsse und Zwischenberichte, eine bevorstehende Zahlungseinstellung oder Überschuldung sowie erhebliche außerordentliche Aufwendungen nach Großschäden" vorsehen würde ${ }^{82}$. Die Art und der Umfang des Investments können jedoch „keinem der dort genannten Regelbeispiele“ zugeordnet werden ${ }^{83}$. Zwar stünde „das fehlende Vorliegen eines der im Emittentenleitfaden genannten Regelbeispiele einer Veröffentlichungspflicht noch nicht prinzipiell entgegen. " ${ }^{84}$ Der Senat kam jedoch zu dem Ergebnis, dass für die Annahme einer Veröffentlichungspflicht keinerlei Anlass bestand, da die Nichtaufführung des Subprime-Anteils im Regelbeispielskatalog des Emittentenleitfadens auch „damals gängiger Auffassung und Einschätzung“ entsprach $^{85}$.

$\mathrm{Zu}$ einem gegenteiligen Ergebnis in dieser Frage kam hingegen der 11. Senat des BGH in der Revisionsinstanz ${ }^{86}$. Zunächst wiederholt der BGH seine bereits zuvor getroffene Feststellung, dass es sich bei dem Emittentenleitfaden lediglich um eine norminterpretierende Verwaltungsvorschrift handele, nur um anschließend auszuführen, dass auch der Emittentenleitfaden den Ausfall wesentlicher Schuldner und erhebliche außerordentliche Aufwendungen zu den veröffentlichungspflichtigen Insiderinformationen zählt und daher auch die Höhe des Subprime-Anteils Kursrelevanz besäße ${ }^{87}$.

\section{cc) Bewertung}

Im Umgang mit dem Emittentenleitfaden der BaFin verdeutlichen beide Fälle ein mittlerweile geändertes Verhaltensmuster der höchstrichterlichen Recht-

81 OLG Düsseldorf v. 7.4.2011, I-6 U 7/10, 6 U 7/10 - juris.

82 OLG Düsseldorf v. 7. 4. 2011, I-6 U 7/10, 6 U 7/10, Rdn. 133 - juris.

83 OLG Düsseldorf v. 7. 4. 2011, I-6 U 7/10, 6 U 7/10, Rdn. 134 - juris.

84 OLG Düsseldorf v. 7. 4. 2011, I-6 U 7/10, 6 U 7/10, Rdn. 135 - juris.

85 OLG Düsseldorf v. 7.4.2011, I-6 U 7/10, 6 U 7/10, Rdn. 135 - juris.

86 BGH, NJW 2012, $1800 \mathrm{ff}-\mathrm{IKB}$.

87 BGH, NJW 2012, 1800, 1805 - IKB. 
sprechung. Zwar wird formal nach wie vor der Emittentenleitfaden mit der bisherigen Ansicht rechtsdogmatisch als norminterpretierende Verwaltungsvorschrift und damit als unverbindliches Innenrecht eingeordnet. Wegen der Unbestimmtheit der Tatbestandsmerkmale des WpHG müssen die Gerichte aber eine umfangreiche Konkretisierung leisten. Gerade bei dem Insiderbegriff des $₫ 13$ Abs. $1 \mathrm{WpHG}$ ist die Tatbestandsoffenheit des Gesetzes mehr als offensichtlich ${ }^{88}$.

Damit setzen sich Gerichte inzwischen auch inhaltlich mit den Bestimmungen des Emittentenleitfadens argumentativ auseinander. Der Emittentenleitfaden erzeugt somit eine Argumentations- bzw. Begründungslast. Entspricht die im Emittentenleitfaden vertretene Auslegung der BaFin nicht der eigenen rechtlichen Beurteilung, so wird das Abweichen teleologisch begründet ${ }^{89}$. Stimmen die Gerichte inhaltlich mit dem Emittentenleitfaden überein, so führen sie ihn an, um ihre rechtliche Bewertung argumentativ zu untermauern ${ }^{90}$. Damit scheint sich in der Rechtsprechung der Obergerichte die Entwicklung abzuzeichnen, die dem Emittentenleitfaden der BaFin eine über die bisherige Meinung hinausgehende Relevanz für die Urteilsfindung beimisst.

\section{Voraussetzungen}

\section{a) Hoheitlicher Rezeptionsakt}

Sekundäre Rechtsquellen bedürfen eines hoheitlichen Rezeptionsaktes ${ }^{91}$, um eine subsidiäre rechtliche Verbindlichkeit erzeugen zu können. So ist anerkannt, dass die Rechnungslegung Standards des DRSC erst vom Bundesministerium der Justiz (BMJ) gem. $\$ 342$ Abs. 2 HGB bekannt gemacht werden müssen, damit sie eine eingeschränkte rechtliche Verbindlichkeit erlangen können ${ }^{92}$. Auch die Empfehlungen der Regierungskommission Corporate Governance sind gem. \161 Abs. 1 Satz 1 AktG vom BMJ im amtlichen Teil des elektronischen Bundesanzeigers zu veröffentlichen. In beiden Fällen findet zuvor zumindest eine eingeschränkte Überprüfung der privaten Standards

88 Klöhn, AG 2012, 345, 348; Möllers/Seidenschwann, NJW 2012, 2762 ff.

89 So die bereits angeführte Vorgehensweise des 20. Senats des OLG Stuttgart zur Frage der Ad-hoc-Relevanz von Zwischenstufen in mehrstufigen Entscheidungsprozessen: OLG Stuttgart, NZG 2009, 624, 626.

90 OLG Düsseldorf v. 7. 4. 2011, I-6 U 7/10, 6 U 7/10 - juris; BGH, NJW 2012, 1800 ff IKB. Zur mittlerweile aufgehobenen Richtlinie gem. \35 Abs. 2 WpHG v. 25. 5.1997, BAnz. 1997, S. 6586: s. BGH, NJW 2006, 1429, 1431.

91 KöNdgen verwendet den Begriff „Inklusionsnorm“, KöNdgen, AcP 206 (2006), 477, $518 \mathrm{ff}$.

92 Ebke/PaAl, Münchener Komm. z. HGB, 2. Aufl., 2008, $\$ 342$ Rdn. 27; Förschle, in: Beck’scher BilanzKomm, 8. Aufl., 2012, $\$ 342$ Rdn. 17. 
statt, um sicher zu stellen, dass die Standards ordnungsgemäß zustande gekommen sind und nicht gegen geltendes Gesetzesrecht verstoßen ${ }^{93}$. Damit sind zumindest formell die Voraussetzungen zur Verhinderung von rechtswidrigen contra legem-Bestimmungen geschaffen ${ }^{94}$. Durch die gesetzgeberische Anerkennung in Rezeptionsakten und die Veröffentlichung durch die Exekutive wird den privaten Standards ein gewisses Maß staatlicher Autorität („auctoritas") vermittelt ${ }^{95}$, die es rechtfertigt, sie rechtquellendogmatisch auf einer höheren Stufe anzusiedeln als rechtlich unverbindliche Kommentarliteratur oder Fachpublikationen. Der staatliche Rezeptionsakt stellt damit ein entscheidendes Kriterium dar, um privaten Standards eine subsidiäre rechtliche Verbindlichkeit als sekundäre Rechtsquelle zuzugestehen ${ }^{96}$.

\section{b) Verfassungsrechtliche Vorgaben- Intra legem-, extra legem-, contra legem-Bestimmungen}

Private Rechtsnormen müssen ein bestimmtes, hinreichendes Niveau demokratischer Legitimation aufweisen, wenn sie eine gewisse Bindungswirkung erzeugen sollen. Der auf dem Rechtsstaatsprinzip beruhende ${ }^{97}$, in Art. 20 Abs. 3 GG aber nicht ausdrücklich genannte Grundsatz vom Gesetzesvorbehalt verlangt, dass bestimmte Maßnahmen des Staates einer parlamentsgesetzlichen Grundlage bedürfen ${ }^{98}$. Auch das Demokratieprinzip des Art. 20 Abs. 2 GG lässt sich zur Begründung dieses Grundsatzes heranziehen: Danach sind wichtige Entscheidungen in öffentlicher Debatte zu klären ${ }^{99}$. Die Rechtsprechung des Bundesverfassungsgerichts fordert, dass der Gesetzgeber in grundlegenden normativen Bereichen alle wesentlichen Entscheidungen selbst treffen muss ${ }^{100}$. Damit darf sich der Gesetzgeber seiner Steuerungsverantwortung nicht vollständig durch Delegation auf andere Normgeber entledigen ${ }^{101}$.

93 Eine Überprüfung auf Zweckmäßigkeit findet hingegen weder bei den Rechnungslegung Standards des DRSC noch bei den Empfehlungen der Regierungskommission Corporate Governance statt: EbKe/PAAL, aaO (Fn. 92), \342 HGB Rdn. 27; Förschle, aaO (Fn. 92), $\$ 342$ HGB Rdn. 17; SpIndler, aaO (Fn. 20), \$161 AktG Rdn. 7; LutTer, aaO (Fn. 20), $\$ 161$ AktG Rdn. 10. S. dazu auch Seibert, BB 2002, 581, 582.

94 Das schließt es nicht aus, dass sich trotz inhaltlicher Überprüfung durch das BMJ einige contra legem-Bestimmungen im DCGK finden, s. dazu unten III.4. und IV.3.b).

95 Zur Bedeutung von Autorität zur Begründung von Rechtsgeltung: RüTHERs/Fischer/ Birk, aaO (Fn. 42) Rdn. 317 ff.

96 Ähnlich Ruffert, aaO (Fn. 46), \$17 Rdn. 89.

97 BVerfGE 78, 179, 197 - Heilpraktikergesetz.

98 BVerfGE 98, 218, 251 - Rechtschreibreform.

99 BVerfGE 85, 386, 403 f; BVerfGE 95, 267, $307 \mathrm{f}$.

100 Zur Wesentlichkeitstheorie, s. etwa: BVerfGE 49, 86, 126 - Kalkar I; BVerfGE 98, 218 Rechtschreibreform, 251 f; hierzu Bauer/C. Möllers, JZ 1999, 696, $700 \mathrm{ff}$.

101 Für die Verweisung auf die Rechnungslegung Standards des DRSC in $\ 342 \mathrm{HGB}, \mathrm{s}$. Berberich, Ein Framework für das DRSC, 2002, S. 137; Номmelhoff/Schwab, aaO 
Um daher ein hinreichend demokratisches Legitimationsniveau bejahen zu können, wird man zwischen den einzelnen Bestimmungen der privaten Regelwerke differenzieren müssen. Als wesentliches Kriterium für die Einstufung als sekundäre Rechtsquelle bietet sich dabei vor allem deren Bezug zum geltenden Recht und somit die bekannte Dreiteilung von Rechtsfindung intra legem, extra legem und contra legem an.

\section{aa) Intra legem-Bestimmungen}

Wenn die „wesentlichen“ politischen Entscheidungen dem parlamentarischen Gesetzgeber vorbehalten bleiben, können private Normsetzer allenfalls konkretisierende Aufgaben in verfassungskonformer Weise wahrnehmen. Durch die gesetzliche Rezeption privater Regelwerke, wie sie etwa durch die Verweisung in $\$ 342 \mathrm{HGB}$ erfolgt, plädiert der Gesetzgeber bewusst für eine Arbeitsteilung zwischen Privaten und Gerichten bei der Konkretisierung von unbestimmten Rechtsbegriffen und Generalklauseln. Die Privaten präzisieren in einem ersten Schritt gesetzliche Regelungen - das Letztentscheidungsrecht bleibt aber den Gerichten vorbehalten ${ }^{102}$. Daher ist auch der teils verwandte Begriff eines „rechtlich abgesicherten Interpretationsmonopols“103 privater Regulierungsgremien so nicht zutreffend, weil ein solches Monopol schon begrifflich andere von der Interpretation ausschließt.

Da diese „Konkretisierung“ dann im Rahmen des Tatbestandes der angewandten Norm stattfindet und diese begründungsmäßig auf das angewandte Recht zurückgeführt werden kann, empfiehlt es sich - in Anlehnung an die Terminologie im Bereich des Richterrechts ${ }^{104}$ - in diesen Fällen von privater Normsetzung intra oder secundum legem zu sprechen. Die privaten Regelsetzer erfüllen die eigentlich den Gerichten zustehende Aufgabe ${ }^{105}$, die durch unbestimmte Rechtsbegriffe und Generalklauseln geprägte Gesetzessprache zu interpretieren und zu konkretisieren und damit für die Praxis operationalisierbar zu machen ${ }^{106}$. Formal betrachtet liegt zwar eine gesetzliche Regelung vor, daher intra legem, die Bewältigung des gesellschaftlichen Problems wurde

(Fn. 33), $\$ 342$ HGB Rdn. 90. Für die Verweisung auf den DCGK in $\$ 161$ AktG: Wernsmann/GatZKa, NZG 2011, 1001, $1005 \mathrm{f} \mathrm{u.} 1007$.

102 Ähnlich Hoнl, der in der Vermutungsregel des $₫ 342$ Abs. 2 HGB eine (teilweise) Verlagerung der Befugnis zur Gesetzeskonkretisierung auf den Deutschen Standardisierungsrat (DSR) und das Bundesministerium der Justiz (BMJ) erblickt, HoHL, aaO (Fn. 18), S. 314; Hellermann, NZG 2000, S. 1097, 1099; siehe auch Berberich, der jedoch davon ausgeht, dass die Befugnis vom Gesetzgeber auf die Exekutive übertragen wurde, BERBERICH, aaO (Fn. 101), S. $136 \mathrm{f}$.

103 Für das DRSC, s. Köndgen, AcP 206 (2006), 477, 490.

104 Gusy, DÖV 1992, 461, 463.

105 S. dazu: P. KirchHof, FS Juristische Fakultät zur 600-Jahr Feier der Univ. Heidelberg, 1986, S. 11, $18 \mathrm{ff}$.

106 Gusy, DÖV 1992, 461, 463. 
aber, oftmals bewusst ${ }^{107}$, an die Rechtsanwendung delegiert ${ }^{108}$. Das Gesetz gibt in diesen Fällen für eine bestimmte Rechtsfrage einen Rahmen vor, dieser bedarf jedoch noch der Ausfüllung und Konkretisierung, um eine bestimmte Rechtsfrage unmittelbar beantworten zu können. Es ist daher ein konkretisierender Zwischenschritt erforderlich, den in diesen Fällen die privaten Regelsetzer übernehmen. Das Gesetz bleibt somit das zentrale Steuerungsinstrument und vermag - gemeinsam mit dem staatlichen Rezeptionsakt - mittelbar eine demokratische Legitimation für die intra legem-Bestimmungen zu vermitteln ${ }^{109}$. In diesem Bereich hat die Regelbildung daher ein hohes Maß an Vermutungswirkung.

\section{bb) Extra legem-Bestimmungen}

Das Gegenstück zur Normsetzung intra legem stellt die private Normsetzung extra legem dar. Sie umfasst all diejenigen Bestimmungen privater Regulierungsgremien, die außerhalb des tatbestandlichen Anwendungsbereichs einer Gesetzesnorm erlassen werden und daher begründungsmäßig nicht auf bereits geltendes Recht zurückgeführt werden können ${ }^{110}$. Sie sind gesetzesübersteigend und formulieren politisch wünschenswerte Zielbestimmungen. Vereinzelt schließen sie Lücken, die der parlamentarische Gesetzgeber oder die Exekutive mangels Rechtsetzungskompetenzen, politischen Normierungswillens oder hinreichender Sachkompetenz bewusst oder unbewusst offen gelassen hat. Hier wiederum erfüllen private Regulierungsgremien Funktionen, die dem klassischen Bereich der Rechtsetzung zugeordnet werden können. Normsetzung extra legem, die mit umfassenden politischen Wertungen verbunden ist, darf der Gesetzgeber nicht auf private Regulierungsgremien delegieren ${ }^{111}$. Der Gesetzgeber kann sich seiner demokratischen Steuerungsverantwortung nicht ohne weiteres entledigen ${ }^{112}$. Weitreichende politische

107 S. dazu unten III.3. und IV.2.b).

108 Kramer, Juristische Methodenlehre, 3. Aufl., 2010, S. 184 f; STERn, Das Staatsrecht der Bundesrepublik Deutschland, Bd. 2, 1980, S. 583.

109 Ähnlich WEBER-REY für „faktisch verpflichtende Regelungssätze“ von Aufsichtsbehörden: „Diese Regelungssätze sind - insofern sie nur gesetzliche Vorgaben konkretisieren und innerhalb einer bestehenden gesetzlichen Ermächtigung entstehen - an den Wortlaut des zu konkretisierenden unmittelbar verpflichtenden Regelungssatzes gebunden, also in ihrer Mittelbarkeit demokratisch legitimiert.“, WeBER-REY, ZGR 2010, 543, 567. S. dazu oben II.1.d).

110 Die Terminologie erfolgt in Anlehnung an Gusy, DÖV 1992, 461, $463 \mathrm{f}$.

$111 \mathrm{Zu}$ weitgehend ist daher der Vorschlag von HavermanN, der dafür eintritt sämtliche Einzelheiten der Bilanz, der Gewinn- und Verlustrechnung und des Anhangs dem DRSC zu überlassen und in Zukunft die Konzernrechnungslegungsvorschriften auf einen einzigen Paragraphen zu beschränken, Havermann, ZGR 2000, 693, $699 \mathrm{f}$.

112 Hommelhoff, FS Odersky, 1996, S. 779, 795; Hellermann, NZG 2000, 1097, 1101; Berberich, aaO (Fn. 101), S. 136, Hommelhoff/Schwab sind daher der Auffassung, dass zentrale Begriffe des Bilanzrechts, wie bspw. das Vollständigkeitsgebot ( $\mathbb{2 4 6}$ 
Entscheidungen privater Standardsetzer, die die Pflichten der Normbetroffenen ausweiten und sich daher als extra legem-Bestimmungen nicht auf eine gesetzliche Regelung zurückführen lassen, können daher schon aus verfassungsrechtlichen Gründen keinerlei rechtliche Verbindlichkeit als sekundäre Rechtsquelle beanspruchen. Damit ist den privaten Standardsetzern eine Rolle als Quasi-(Ersatz-)Gesetzgeber verschlossen. Diese These entspricht auch weitestgehend der herrschenden Meinung, die den privaten Regulierungsgremien das Recht abspricht, gesetzesübersteigende Bestimmungen mit rechtlicher Wirkung zu erlassen ${ }^{113}$. Gerade die fehlende Rückbindung an das Gesetz sorgt dafür, dass private Normsetzung extra legem lediglich als Rechtserkenntnisquelle eingestuft werden kann.

\section{cc) Contra legem-Bestimmungen}

Schließlich muss eine Vermutungswirkung Bestimmungen versagt bleiben, die von den privaten Gremien contra legem erlassen wurden. Normsetzung contra legem liegt etwa dann vor, wenn Regelungen sich zwar innerhalb des tatbestandlichen Anwendungsbereiches einer gesetzlichen Regelung befinden, mit dem telos der Norm jedoch inhaltlich schlechterdings unvereinbar sind. Sie liegt auch dann vor, wenn diese Bestimmungen nicht im Einklang mit der Verfassung oder mit sachähnlichen Parlamentsgesetzen und Rechtsverordnungen stünden ${ }^{114}$.

\section{Rechtsfolgen}

In Anlehnung an das Richterrecht lassen sich auch bei den Empfehlungen des DCGK und den Deutschen Rechnungslegung Standards als sekundäre Rechtsquellen eine Befassungs- und subsidiäre Befolgungspflicht begründen.

Abs. 1 Satz 1 HGB) und der Einzelbewertungsgrundsatz ( $\$ 252$ Abs. 1 Nr. 2 HGB) vom Gesetzgeber selbst definiert werden müssen, Номмецноғғ/Sснwав, аaО (Fn. 33), $\$ 342$ HGB Rdn. 91; P. KirchHoF, Handbuch des Staatsrechts, 3. Aufl., 2007, $§ 99$ Rdn. 134.

113 Für das DRSC: Merkt, in: Baumbach/Hopt, 35. Aufl., 2012, \342 Rdn. 2; HaverMANN, ZGR 2000, 693, 696; Für die Regierungskommission Corporate Governance: Ringleb, in: Ringleb/Cremer/Lutter/v. Werder, DCGK, 4. Aufl., 2010, Rdn. 17 u. 20. A. A. LutTer, FS Forstmoser, 2003, S. $287 \mathrm{ff}$.

114 Vgl. zur parallelen Fragestellung im Bereich der judikativen Normkonkretisierung: Röthel, Normkonkretisierung im Privatrecht, 2004, S.113; Möllers, FS G. Roth, 2011, S. 474, 478, $487 \mathrm{f}$. 


\section{a) Befassungspflicht}

Im Einzelnen müssen sich die einschlägigen Instanzen, wie etwa die Rechtsprechung, mit den sekundären Rechtsquellen befassen. Da es sich bei den sekundären Rechtsquellen um normative Regelwerke und nicht um Tatsachen handelt, trifft die Parteien diesbezüglich keine Behauptungslast. Vielmehr wird der Grundsatz „iura novit curia" 115 auch auf sekundäre Rechtsquellen auszudehnen sein. Dies ist bei Rechtserkenntnisquellen, wie der Rechtsliteratur oder ausländischem Recht, dagegen nicht der Fall.

\section{b) Subsidiäre Befolgungspflicht}

Bei den Verweisungen normiert der Gesetzgeber regelmäßig, dass zugunsten des Normunterworfenen die widerlegbare Vermutung gesetzeskonformen Handelns gilt: Soweit sich der Betroffene an die privaten Regelwerke hält, wird widerlegbar vermutet, dass er rechtmäßig gehandelt hat. Bei den normkonkretisierenden Verweisungen ist anerkannt, dass die privaten Regelwerke den Stand der (Wissenschaft und) Technik mit der widerlegbaren Vermutung der Richtigkeit ${ }^{116}$ umschreiben. Vergleichbare Vermutungswirkungen finden sich im europäischen Recht ${ }^{117}$ und im internationalen Wirtschaftsrecht ${ }^{118}$. Es handelt sich dabei nicht um Beweiserleichterungen, da nur Tatsachen dem Beweis zugänglich sind. Vielmehr helfen sie dem Rechtsanwender über die Unsicherheit bei der Auslegung und Konkretisierung von unbestimmten Rechtsbegriffen und Generalklauseln hinweg. Im Gegensatz zu Rechts- und Tatsachenvermutungen bieten sekundäre Rechtsquellen einen rechtlichen Ausweg für eine rechtliche, nicht tatsächliche Unsicherheit ${ }^{119}$. Diese widerlegbare Vermutung können somit nur Bestimmungen entfalten, die sich innerhalb des tatbestandlichen Anwendungsbereiches eines Gesetzes (intra legem) befinden. Eine Vermutungswirkung greift hingegen nicht ein, wenn die private Rechtsetzung außerhalb des Gesetzes erfolgt, wie dies bei einer Regelsetzung extra legem oder contra legem der Fall ist.

115 „Das Gericht kennt das Recht.“ S. dazu: Meder, Ius non scriptum - Traditionen privater Rechtsetzung, 2. Aufl., 2009, S. 186 f; Rauscher, Münchener Komm. z. ZPO, 3. Aufl., 2008, Einl. Rdn. 306.

116 Marburger, aaO (Fn. 6), S. 395 ff; Ruffert, aaO (Fn. 46), $\$ 17$ Rdn. 89.

117 S. Neue Konzeption auf dem Gebiet der technischen Harmonisierung und Normung v. 7.5.1985, ABl. 1985, Nr. C 136, S. 2 f. Hierzu Marburger, FS Feldhaus, 1999, S. 387, 392.

118 Für das WTO-Recht s. Art. 2.5. S. 2 Technical Barriers to Trade (TBT)-Übereinkommen v. 23.12.1994, ABl. Nr. L 336, S. 86.

$119 \mathrm{Zu}$ Rechts- und Tatsachenvermutungen ausführlich: Косн, Reichweite und Grenzen der Mitwirkungsverantwortung der Parteien des Zivilprozesses sowie der Darlegungsund Beweisanforderungen, 2012, S. 413, $419 \mathrm{ff}$. 
Das Gericht trifft dabei gem. $\$ 139$ ZPO die prozessuale Pflicht, die Parteien auf die Vermutungswirkung der sekundären Rechtsquelle hinzuweisen. Damit hat die Partei, zu deren Lasten die Vermutung wirkt, die Möglichkeit, durch eine ausführliche Gegenargumentation die Entscheidung des Richters zu beeinflussen. Das Gericht muss die Argumentation der Partei gelten lassen, wenn diese vorträgt, dass die sekundäre Rechtsquelle entweder auf überholten wissenschaftlichen Erkenntnissen beruht oder aufgrund der besonderen Umstände des konkreten Einzelfalls mit dem „telos“ der gesetzlichen Bestimmungen unvereinbar ist. Die vorgetragene Lösung muss der Rechtsordnung jedoch deutlich besser entsprechen als die sekundäre Rechtsquelle ${ }^{120}$. Der Umstand, dass sie lediglich gleich gut vertretbar ist, ist daher nicht ausreichend ${ }^{121}$. Die „Unrichtigkeit“ der intra legem-Bestimmung muss sich dem Richter aufgrund der Argumentation der Partei aufdrängen.

Die subsidiäre Verbindlichkeit sekundärer Rechtsquellen wird sich daher vornehmlich im „non liquet“ Bereich auswirken ${ }^{122}$. Sieht sich der Richter bei der Auslegung und Konkretisierung von Rechtsnormen vor zwei Auslegungsalternativen gestellt, die er beide für gut begründbar hält, so hat er die intra legem Regelsetzung zu beachten und eine Abweichung davon zu begrün$\operatorname{den}^{123}$.

Auch besteht im Rahmen der comply-or-explain-Regelung des $\$ 161 \mathrm{AktG}$ die Möglichkeit, der Befolgung einzelner Empfehlungen des DCGK-Regelwerkes zu widersprechen. Mit einem ausführlich begründeten Widerspruch kann das Unternehmen versuchen, die Vermutungswirkung vor Gericht zu widerlegen ${ }^{124}$.

\section{Deutsche Rechnungslegung Standard}

\section{1. $\int 342 H G B$ als boheitlicher Rezeptionsakt}

Die Rechnungslegung Standard des DRSC lassen sich als Beispiel für eine sekundäre Rechtsquelle anführen. Der Gesetzgeber bedient sich durch die Verweisung in $\$ 342$ HGB privaten Sachverstandes auf dem Gebiet des Konzernrechnungslegungsrechts und gesteht in $\$ 342$ Abs. 2 HGB den erarbeiteten Rechnungslegung Standard ausdrücklich die für sekundäre Rechtsquellen

120 Für Präjudizien: Bydlinski, Juristische Methodenlehre und Rechtsbegriff, 2. Aufl., 1991, S. 507 f.

121 Bydlinski, aaO (Fn. 120), S. 507 f; DERs., JZ 1985, 149, 151.

122 Bydlinski, aaO (Fn. 120), S. 506; DERs., JZ 1985, 149, 152.

123 Bydlinski, aaO (Fn. 120), S. 506. S. dazu auch: Langenbucher, aaO (Fn. 50), S. 119.

124 Für eine kurze und prägnante Begründung hingegen: MülbeRT, aaO (Fn. 26), S. 14; Ringleb, aaO (Fn. 113), Rdn. 1568. S. unten IV.5. b). 
charakteristische subsidiäre Verbindlichkeit zu. $\$ 342$ Abs. 2 HGB enthält eine sog. normative Konformitätsvermutung ${ }^{125}$. Die gem. $\ 342$ Abs. 1 HGB erarbeiteten und vom BMJ bekannt gemachten Standards haben nach dem Gesetz die Vermutung für sich, Grundsätze ordnungsgemäßer Buchführung im Rahmen der Konzernrechnungslegung zu sein ${ }^{126}$. Die Gerichte sind daher zunächst einmal verpflichtet, von einer Konformität des Konzernabschlusses mit den Grundsätzen ordnungsgemäßer Buchführung auszugehen.

\section{Der Inbalt der Vermutungswirkung}

Vom rechtsdogmatischen Standpunkt her werden diese normativen Konformitätsvermutungen in der Literatur als ein Sonderfall betrachtet. Denn während bei gesetzlichen Tatsachenvermutungen ${ }^{127}$, bei Vorliegen der Vermutungsbasis auf eine Tatsache (Vermutungsinhalt) und bei gesetzlichen Rechtsvermutungen ${ }^{128}$, bei Erfüllung der Vermutungsbasis auf einen bestimmten Rechtszustand (Vermutungsinhalt) geschlossen wird, verhält es sich bei normativen Konformitätsvermutungen anders. In diesen Fällen stellen die privaten Regelwerke die Vermutungsgrundlage dar, mit deren Hilfe ein unbestimmter Rechtsbegriff konkretisiert wird ${ }^{129}$. Im Kern geht es daher um Normkonkretisierung unter Zuhilfenahme von privatem Sachverstand ${ }^{130}$. Es handelt sich daher nicht um Beweisregeln im prozessualen Sinne, da bei der hier in Frage stehenden Materie normative Wertungen eine erhebliche Rolle spielen ${ }^{131}$.

Durch die Normierung eines Vermutungstatbestandes hat der Gesetzgeber deutlich gemacht, dass er das in Fragen der Interpretation und Konkretisierung von Gesetzesbestimmungen bestehende Konkurrenzverhältnis zwischen privaten Regulierungsgremien und Gerichten zu Gunsten der Privaten entschieden hat ${ }^{132}$.

$125 \rrbracket 342$ Abs. 2 HGB lautet: „Die Beachtung der die Konzernrechnungslegung betreffenden Grundsätze ordnungsmäßiger Buchführung wird vermutet, soweit vom Bundesministerium der Justiz bekannt gemachte Empfehlungen einer nach Absatz 1 Satz 1 anerkannten Einrichtung beachtet worden sind.“

126 Ringleb, aaO (Fn. 113), Rdn. 56.

127 Typische Beispiele sind: $\mathbb{\int} \int 363,685$ Abs. 2, 938, 1117 Abs. 3, 1213 Abs. 2, 1253 Abs. 2, 1377 Abs. 3, 1600, 1600 o Abs. 2 Satz 1, 1625, 2009, 2270 Abs. 2 BGB.

128 Typische Beispiele sind: $\$ \int 891,921,1006,1138,1155,1360$ b, 1362, 1380 Abs. 1 Satz 2, 1620, 1964, 2255 S. 2, 2365 BGB.

129 So auch: Евке, ZIP 1999, 1193, 1202.

130 Aus diesem Grunde lehnen Номmelhoff/Schwaв den Begriff Vermutung gänzlich ab: Hommelhoff/Schwab, aaO (Fn. 33), \342 HGB Rdn. 84. Ebke hingegen spricht von Vermutung mit materiell-rechtlichem Gehalt: EвкE, ZIP 1999, 1193, 1202.

131 Budde/STEuber, DStR 1998, 1181, 1184.

132 Hellermann, NZG 2000, 1097, 1099; Berberich, aaO (Fn. 101), S. 136; Hohl, aaO (Fn. 18), S. 314; Augsberg, aaO (Fn. 10), S. 193. 


\section{Normsetzende Standards intra legem}

Das Deutsche Rechnungslegungs Standard Commitee soll gem. $\$ 342$ Abs. 1 HGB gemeinsam mit dem Bundesministerium der Justiz die Grundsätze der ordnungsgemäßen Konzernrechnungslegung (GoK) weiterentwickeln. Dazu zählen auch die Grundsätze ordnungsgemäßer Buchführung (GoB), welche gem. $\$ 297$ Abs. 2 HGB auch im Konzernabschluss anzuwenden sind. Die Rechnungslegungsstandards des DSR erfüllen primär die Funktion, diese unbestimmten Rechtsbegriffe zu konkretisieren.

\297 Abs. 1 Satz 1 HGB bestimmt etwa, dass der Konzernabschluss eine Kapitalflussrechnung enthalten muss. Optional besteht seit dem BilReG ${ }^{133}$ für Mutterunternehmen die Möglichkeit, den Konzernabschluss um eine Segmentberichterstattung zu erweitern, $\$ 297$ Abs. 1 Satz 2 HGB. Welche inhaltlichen Anforderungen an die Kapitalflussrechnung und die Segmentberichterstattung zu stellen sind, werden jedoch von den gesetzlichen Bestimmungen nicht vorgegeben ${ }^{134}$. Der parlamentarische Gesetzgeber hat bewusst darauf verzichtet, die einzelnen Voraussetzungen der Kapitalflussrechnung bzw. der Segmentberichterstattung gesetzlich zu umschreiben ${ }^{135}$. So heißt es in den Gesetzgebungsmaterialien:

„Es wird dabei bewusst darauf verzichtet, gesetzlich die einzelnen Voraussetzungen der Kapitalflussrechnung bzw. Segmentberichterstattung zu umschreiben. Dies könnte (...) eine Aufgabe des Rechnungslegungsgremiums sein. "136

In Erfüllung dieser ihm übertragenen Aufgabe veröffentlichte das DRSC daraufhin die Deutschen Rechnungslegungsstandards Nr. 2 und Nr. 3, welche die Anforderungen an die Konzernrechnungslegung börsennotierter Mutterunternehmen en détail vorgeben ${ }^{137}$. Mit der konkretisierenden Normsetzung intra legem findet eine Rückbindung an das parlamentarische Gesetz statt. Die Vermutungswirkung des $\$ 342 \mathrm{Abs} .2 \mathrm{HGB}$ ist deshalb gerechtfertigt, so dass intra legem erlassene Rechnungslegungsstandards nachvollziehbar als sekundäre Rechtsquellen einzustufen sind.

133 Gesetz zur Einführung internationaler Rechnungslegungsstandards und zur Sicherung der Qualität der Abschlussprüfung (Bilanzrechtsreformgesetz-BilReG) vom 4.12. 2004, BGBl. I (2004), S. 3166.

134 Wiedmann, in: Ebenroth/Boujong/Joost/Strohn, HGB, 2. Aufl., 2008, $\$ 342$ Rdn. 8; MerkT, aaO (Fn. 113), \$297 HGB Rdn. 1.

135 BT-Drs. $13 / 10038$ v. 4.3.1998, S. 26; v. Wysocki, DB 1999, 2373; Pilhofer, DStR 2000, 292; Baetge/Krumnow/Noelle, DB 2001, 769, 770.

136 BT-Drs. 13/10038 v. 4.3.1998, S. 26.

137 Havermann, ZGR 2000, 693, 699; Die Deutschen Rechnungslegungsstandards Nr. 2 und Nr. 3 werden noch ergänzt durch die DRS 2-10, 2-20, 3-10, 3-20, die besondere Voraussetzungen für Kreditinstitute und Versicherungsunternehmen aufstellen. Die jeweiligen Fundstellen finden sich unter www.standardsetter.de. 


\section{Feblende Vermutungswirkung bei normsetzenden Standards extra legem und contra legem}

Es finden sich jedoch auch Bestimmungen des Rechnungslegungsgremiums, die unabhängig von gesetzlichen Vorgaben gänzlich neue Anforderungen formulieren und daher der Normsetzung extra legem zuzurechnen sind: DRS 6 sieht in Anm. 11 für Unternehmen die Verpflichtung vor, jeweils zum Stichtag der ersten drei Quartale einen Zwischenbericht zu veröffentlichen. Eine gesetzliche Grundlage für eine solche Verpflichtung gibt es nicht ${ }^{138}$. Als weitere über das Gesetz hinausgehende Bestimmung zählt auch DRS 4 Anm. 56, der im Falle eines Unternehmenskaufes das erwerbende Unternehmen dazu verpflichtet, den Gewinn je Aktie (earnings per share) im Anhang der Bilanz auszuweisen. Auch dafür gibt es keine korrespondierende Vorschrift im HGB $^{139}$.

Solchen Bestimmungen muss eine Vermutungswirkung versagt bleiben, da es hier an einer hinreichenden demokratischen Legitimation für den Erlass von gesetzesübersteigenden Standards fehlt ${ }^{140}$. In die gleiche Kategorie fallen Bestimmungen, die gesetzliche Wahlrechte einschränken. Wird durch Rechnungslegungsstandards ein gesetzgeberisch eingeräumter Entscheidungsspielraum eingeschränkt, so erfolgt die Normsetzung contra legem. Hier scheidet eine Vermutungswirkung schon deshalb aus, weil ein privates Rechnungslegungsgremium gesetzliche Regelungen nicht außer Kraft setzen kann ${ }^{141}$. Ein Beispiel für eine solche Einschränkung von Wahlrechten war die Behandlung des Geschäfts- oder Firmenwerts. Nach $\$ 309$ Abs. 1 Satz 3 HGB a.F war sowohl die erfolgsneutrale als auch die erfolgswirksame Abschreibung gesetzlich zulässig ${ }^{142}$. DRS 4 Anm. 31 schränkte diese Wahlrechte jedoch ein und ließ nur die planmäßige lineare Abschreibung des Geschäfts- oder Firmenwerts über die geplante Nutzungsdauer $\mathrm{zu}^{143}$.

138 Aus diesem Grunde schlägt Wiedmann nur dann eine Verbindlichkeit der Bestimmung vor, wenn die Unternehmen aus anderen Gründen verpflichtet sind, Zwischenberichte aufzustellen, bspw. bei Vereinbarungen mit der Deutschen Börse, WiedmanN, aaO (Fn. 134), \342 HGB Rdn. 10.

139 Förschle, aaO (Fn. 92), \342 HGB Rdn. 19.

140 Merkt, aaO (Fn. 113), \342 HGB Rdn. 2; weitergehend: Köndgen, AcP 206 (2006), 477, $490 \mathrm{f}$, der dem DRS neben einem rechtlich abgesicherten Interpretationsmonopol auch ein Rechtsfortbildungsmonopol zugestehen möchte.

141 Wiedmann, aaO (Fn. 134), $\$ 342$ HGB Rdn. 9; Psaroudakis, in: Heidel/Schall, HGB, 2011, $\$ 342$ Rdn. 8.

142 So auch zur alten Fassung s. Merkt, aaO (Fn. 113), $\$ 309$ HGB Rdn. 1.

143 Zur alten Fassung: Förschle, aaO (Fn. 92), \342 HGB Rdn. 9. 
Konsequenterweise wurden diese Regelungen inzwischen auch aufgehoben ${ }^{144}$. Im Ergebnis beschränkt sich damit die Vermutungswirkung auf die private Rechtssetzung intra legem ${ }^{145}$.

\section{Widerlegung der Vermutungswirkung} bei nicht überzengenden Empfeblungen

Eine abweichende Handhabung von den Empfehlungen des DSRC ist daher nur zulässig, wenn die Partei, zu deren Ungunsten die Vermutung gilt, nachweist, dass die empfohlene Handhabung nicht geeignet ist, die gesetzlichen Ziele zu verwirklichen und deshalb die abweichende Verfahrensweise gewählt wurde, um den Grundsätzen ordnungsgemäßer Konzernrechnungslegung zu entsprechen ${ }^{146}$. Ob es der Partei gelungen ist die Vermutung zu widerlegen, entscheidet das Gericht. Es muss zu der Überzeugung gelangen, dass die Empfehlungen im konkreten Fall nicht zu einer richtigen, gesetzmäßigen und damit ordnungsgemäßen Konzernbilanzierung führen ${ }^{147}$.

Erst wenn dargelegt wird, dass der Sachverhalt atypisch ist und von den Empfehlungen der Rechnungslegungsstandards nicht erfasst wird oder die Standards auf wissenschaftlich überholten Erkenntnisgrundlagen beruhen, fällt den Gerichten wieder die Aufgabe zu, den unbestimmten Rechtsbegriff der GoB - notfalls unter Zuhilfenahme von Sachverständigen - zu konkretisie$\operatorname{ren}^{148}$.

144 Gegenwärtig werden keinerlei gesetzliche Wahlrechte durch die DRS eingeschränkt, Förschle, aaO (Fn. 92), $\$ 342$ HGB Rdn. 19.

145 Für eine Einschränkung der Vermutungswirkung auf gesetzesauslegende und konkretisierende Rechnungslegungsstandards auch: WiedmanN, aaO (Fn. 134), $\$ 342 \mathrm{HGB}$ Rdn. 8 f; Ebke/Paal, aaO (Fn. 92), $\$ 342$ HGB Rdn. 25; Psaroudakis, aaO (Fn. 141), \342 HGB Rdn. 8; Merkt, aaO (Fn. 113), \342 HGB Rdn. 2. Ähnliche Einteilung auch bei Förschle, aaO (Fn. 92), $\$ 342$ HGB Rdn. 19.

146 So die Selbsteinschätzung des DRSC im Jahre 2002, Deutsches Rechnungslegungs Standards Committee e.V., Funktion, Aufgaben und Projekte, S.18, abrufbar unter: http://www2.wiwi.hu-berlin.de/institute/rewe/download/forum/bumboldt_drsc.pdf.

147 Förschle, aaO (Fn. 92), \$342 HGB Rdn. 19; Ernst, WPg, 1998, 1031; Ebke/PaAl, aaO (Fn. 92), $\$ 342$ HGB Rdn. 24.

148 Berberich, aaO (Fn. 101), S. 135; Eine vollständige rechtliche Bindung aufgrund des unverhältnismäßigen, prohibitiv hohen Aufwands daher bejahend: HommelHoff/ Schwab, aaO (Fn. 33), \342 HGB Rdn. 82; Eifert, aaO (Fn. 8), \$19 Rdn. 65. 


\section{Deutscher Corporate Governance Kodex}

\section{1. $\int 161 \mathrm{Akt} G$ als boheitlicher Rezeptionsakt}

Auch die gesetzliche Pflicht zur Abgabe einer Entsprechenserklärung nach dem comply-or-explain-Prinzip ( $\$ 161$ Abs. 1 AktG) ist eine Form der hoheitlichen Rezeption privater Regelwerke ${ }^{149}$. Der Gesetzgeber verpflichtet den Vorstand und den Aufsichtsrat einer börsennotierten Gesellschaft den Aktionären zu erklären, ob sie den Empfehlungen der Regierungskommission Deutscher Corporate Governance Kodex ganz, nur teilweise oder überhaupt nicht entsprochen haben, und falls nicht, diese Abweichung zu begründen ${ }^{150}$. Insofern wird daher auch von einem Geltungsanspruch mit Ausstiegsklausel gesprochen ${ }^{151}$. Die opt-out-Alternative eröffnet den Unternehmen die Möglichkeit negativ zu erklären, dass einzelne Bestimmungen des Kodex für sie nicht gelten sollen, weil sie mit den spezifischen Eigenheiten des Unternehmens ihrer Auffassung nach nicht vereinbar sind $^{152}$.

Der Gesetzgeber hat sich bei den Empfehlungen der Regierungskommission gegen eine normative Konformitätsvermutung, wie im Falle der Rechnungslegungsstandards, entschieden ${ }^{153}$. Nach der hier vertretenden Ansicht reicht jedoch die hoheitliche Rezeption der Empfehlungen aus, um den intra legemBestimmungen der Regierungskommission eine staatliche Autorität zu verleihen und ihnen damit eine subsidiäre rechtliche Verbindlichkeit als sekundäre Rechtsquelle zuzugestehen ${ }^{154}$. Anregungen wird man hingegen eine solche Wirkung nicht zugestehen können, da sie rechtspolitische Wunschvorstellungen der Regierungskommission enthalten und daher über das Gesetz hinausgehen ${ }^{155}$. Auch dem rechtsbeschreibenden Teil des Kodex wird man eine Bindungswirkung nicht zusprechen können. Denn dort, wo der Kodex das Gesetz zutreffend wiedergibt, ergibt sich die Bindungswirkung bereits

149 Hohl, aaO (Fn. 18), S. 37 ff; hierzu Möllers/Hailer, JZ 2012, 841, 843.

150 Durch das Gesetz zur Modernisierung des Bilanzrechts (BilMoG) v. 25. 5. 2009 (BGBl. I, 1102) wurde die bisherige Pflicht zur Offenlegung von Abweichungen durch eine Begründungspflicht ersetzt. Im Einzelnen dazu: WeIss, aaO (Fn. 32), S. 82 f.

151 Hüffer, aaO (Fn. 35), $\$ 161$ AktG Rdn. 3.

152 Präambel des Deutschen Corporate Governance Kodex in der Fassung vom 15. 5.2012; Begr. RegE BT-Drs. 14/8769, S.21; SpIndler, aaO (Fn. 20), $\$ 161$ AktG Rdn. 38 .

153 Aus diesem Grunde wird in der Literatur teilweise darauf geschlossen, dass die KodexEmpfehlungen eine „niedrigere Normhöhe“ hätten: Ringleb, aaO (Fn. 113), Rdn. 63; kritisch zu dieser Einschätzung: WeIss, aaO (Fn.32), S.112f („Gleichartigkeit der Situation").

154 S. oben II.2.a).

155 v. Werder, in: Ringleb/Cremer/Lutter/v. Werder, DCGK, 4. Aufl., 2010, Rdn. 125 („proaktive Vorstöße für die weitere Entwicklung der Corporate Governance“). 
unmittelbar aus der gesetzlichen Regelung. In den Fällen, in denen das Gesetz verfälscht und damit contra legem wiedergegeben wird, verstößt eine solche Kodexbestimmung gegen höherrangiges Recht ${ }^{156}$.

\section{Heranziehung des klassischen Auslegungskanons zur Bestimmung der Bindungswirkung im Bereich von intra legem-Regeln des DCGK}

Ob eine Empfehlung sich an den gesetzlichen Vorgaben orientiert und damit intra legem ergeht, ist durch Auslegung zu ermitteln. Dazu lässt sich der klassische Auslegungskanon, also Wortlaut, historischer Wille, Systematik und Telos nutzbar machen.

\section{a) Wortlaut}

Eine Bindungswirkung von Kodexbestimmungen lässt sich umso leichter begründen, je stärker sich diese deduktiv vom Wortlaut einer gesetzlichen Regelung ableiten lassen. Das Gesetz bildet dabei den Rahmen, innerhalb dessen mehrere Möglichkeiten der Auslegung und Konkretisierung gegeben sind ${ }^{157}$. Je besser sich die Kodexbestimmung innerhalb dieses vom Gesetzgeber vorgegebenen Rahmens verorten lässt, umso eher lässt sich auch eine eingeschränkte Bindungswirkung für die Gerichte argumentativ begründen. Denn in diesem Fall gibt es eine mittelbare, legitimationsstiftende Rückbindung der Kodexempfehlungen an das parlamentarische Gesetz ${ }^{158}$. Den vom Gesetzgeber verwendeten Begrifflichkeiten kann somit eine entscheidende Bedeutung bei der Frage zukommen, inwieweit Kodexbestimmungen als sekundäre Rechtsquelle qualifiziert werden können. In Anlehnung an Modelle, die in der juristischen Methodenlehre für den Bereich der grammatischen Auslegung entwickelt wurden ${ }^{159}$, wird man zwischen Kodexbestimmungen unterscheiden müssen, die als Interpretationen eindeutig einem bestimmten gesetzlichen Begriff zugeordnet werden können, Bestimmungen, bei denen eine Zuordnung aufgrund der zunehmenden Unbestimmtheit des gesetzlichen Wortlauts

156 S. dazu unten IV.3.b). Aus diesem Grunde sollte der rechtsbeschreibende Teil aus dem DCGK herausgenommen werden, vgl. Habersack, Gutachten DJT, 2012, E 57.

157 Kelsen, Reine Rechtslehre, 2. Aufl., 1967, S. 348.

158 S. dazu bereits oben II.2.b)aa).

159 Das wohl bekannteste Modell stellt in diesem Zusammenhang die von Heck begründete Unterscheidung in Begriffskern und Begriffshof dar, Неск, AcP 112 (1914), 1, $121 \mathrm{ff} ;$ Косн befürwortet ein Drei-Bereiche-Modell. Er unterscheidet dabei zwischen Begriffen, auf die der fragliche Ausdruck eindeutig angewendet (positiv) oder eindeutig abgelehnt (negativ) werden kann. Eine dritte Gruppe bilden die neutralen Begriffe, bei denen eine semantische Zuordnung nicht eindeutig möglich ist, vgl. KocH, ARSP 61 (1975), 27, 34; DERs./RüssmanN, Juristische Begründungslehre, 1982, S. 194 ff. 
nicht eindeutig möglich ist, und Kodexbestimmungen, die zweifelsfrei nicht einer bereits bestehenden gesetzlichen Regelung zugeordnet werden können.

So wurde im Zuge der Neufassung des $\ 87$ Abs. 1 AktG durch das VorstAG im Jahre $2009^{160}$ die „übliche Vergütung“ als Bemessungskriterium in das Gesetz eingefügt. Die Kodexempfehlung konkretisiert diesen unbestimmten Rechtsbegriff in Ziff. 4.2.2. Abs. 2 dahingehend, dass nicht nur die Branchenüblichkeit (horizontaler Vergleich) sondern auch die Vergütungsstruktur innerhalb der Gesellschaft (vertikaler Vergleich) berücksichtigt werden soll ${ }^{161}$.

\section{b) Gesetzgebungsgeschichte}

Ergibt die historisch-genetische Auslegung, dass der Gesetzgeber ausweislich der Gesetzgebungsmaterialen einzelnen Kodexbestimmungen eine herausragende Bedeutung bei der Auslegung und Konkretisierung vom Gesetzesrecht oder gar eine lückenausfüllende Funktion beimisst, so ist ein solcher Bezug des Gesetzgebers zwar noch keine Verweisungsnorm mit Vermutungswirkung. Es ist aber ein weiteres Indiz des historischen Gesetzgebers für die Relevanz des privaten Normgebers und kann in Zusammenspiel mit anderen Kriterien zu der oben genannten Befassungs- und Befolgungspflicht führen ${ }^{162}$. Eine Inbezugnahme einer Kodexempfehlung findet sich in den Gesetzgebungsmaterialien des Gesetzes über die Offenlegung von Vorstandsvergütungen ${ }^{163}$. In $\ 285 \mathrm{Nr} .9 \mathrm{HGB}$ verpflichtete der Gesetzgeber die Gesellschaften dazu, die Gesamtbezüge ihrer Aufsichtsratsmitglieder im Anhang des Jahresabschlusses anzugeben. Hinsichtlich einer individualisierten Offenlegung der Aufsichtsratvergütung verweist der Gesetzgeber jedoch auf die in Ziff. 5.4.5 (nunmehr Ziff. 5.4.6 des Deutschen Corporate Governance Kodex i. d. F. vom 26.5.2010) enthaltene Empfehlung der Regierungskommission:

„Zur Aufsichtsratsvergütung scheint es daher ausreichend, eine entsprechende Verpflichtung zur Individualangabe wie bisher ausschließlich im Corporate

160 Gesetz zur Angemessenheit der Vorstandsvergütung (VorstAG) v. 31.7.2009, BGB1. I 2509.

161 S. dazu auch Kling, DZWIR 2010, 221, 225. Hinzu kommt, dass der Kodex mit diesem Kriterium eine Interpretation aufgreift, die bereits in der Regierungsbegründung enthalten war, RegE VorstAG, BT-Drs. 16/13433, S. 10.

162 Ähnlich: LeYENs, aaO (Fn. 17), $\$ 161$ AktG Rdn. 102 („Ausstrahlungswirkung“); Zur Rolle des historischen Argumentes s. BVerfGE 122, 248 - Rügeverkümmerung. RüTHers/Fischer/Birk, aaO (Fn. 42), Rdn. 799; RüThers, NJW 2009, 1461 ff; CH. MöLLERS, JZ 2009, $668 \mathrm{ff}$.

163 RegE eines Gesetzes über die Offenlegung der Vorstandsvergütungen (Vorstandsvergütungs-Offenlegungsgesetz - VorstOG), BT-Drs. 15/5577. 
Governance Kodex unter 5.4.5 vorzusehen und auf gesetzliche Regelungen zu verzichten. “"164

Der Kodexempfehlung in Ziff. 5.4.6. kommt daher ausweislich der Gesetzgebungsmaterialien eine lückenausfüllende, gesetzesvertretende Funktion zu. Ein weiteres Beispiel findet sich in der Regierungsbegründung des Gesetzes zur Modernisierung des Bilanzrechts ${ }^{165}$. Nach den $\$ \mathbb{S} 100$ Abs. 5, 107 Abs. 4 AktG muss mindestens ein unabhängiges Mitglied des Aufsichtsrats bzw. des Prüfungsausschusses über Sachverstand auf dem Gebiet der Rechnungslegung oder der Abschlussprüfung verfügen (sog. unabhängiger Finanzexperte). Der Gesetzgeber verzichtete dabei bewusst darauf, das Tatbestandsmerkmal der Unabhängigkeit zu definieren und verweist u. a. auf den DCGK ${ }^{166}$ :

„Nach dem Deutschen Corporate Governance Kodex in der Fassung vom 14. Juni 2007 ist ein Aufsichtsratsmitglied als unabhängig anzusehen, wenn es in keiner geschäftlichen oder persönlichen Beziehung zu der Gesellschaft oder deren Vorstand steht, die einen Interessenkonflikt begründet (Ziffer 5.4.2 Satz 2)." ${ }^{167}$

Der Gesetzgeber spricht davon, dass der DCGK „Hinweise [gibt] auf Aspekte, die für die Beurteilung der Unabhängigkeit eines sachverständigen Aufsichtsratsmitglieds eine Rolle spielen können. "168 In diesem Fall gibt der Gesetzgeber dem Rechtsanwender und damit auch den Gerichten die Vorgabe, sich mit der entsprechenden Kodexempfehlung zunächst einmal zu befassen.

164 RegE VorstOG, BT-Drs. 15/5577, S. 11.

165 RegE Gesetz zur Modernisierung des Bilanzrechts (Bilanzrechtsmodernisierungsgesetz - BilMoG), BT-Drs. 16/10067. Das Gesetz diente der Umsetzung der Richtlinie 2006/43 EG des Europäischen Parlaments und des Rates über Abschlussprüfungen von Jahresabschlüssen und konsolidierten Abschlüssen, zur Änderung der Richtlinien 78/660/EWG und 83/349/EWG des Rates und zur Aufhebung der Richtlinie 84/253/EWG des Rates v. 17.5.2006, ABl. Nr.L 157, S. 87 (Abschlussprüfer-RiL 2006/43 EG).

166 RegE BilMoG, S. 101 f; Hüffer, aaO (Fn. 35), \$100 AktG Rdn. 12. In der Regierungsbegründung wird des Weiteren auf die Empfehlung der EU-Kommission v. 15. 2. 2005 zu den Aufgaben von nicht geschäftsführenden Direktoren/Aufsichtsratsmitgliedern börsennotierter Gesellschaften sowie zu den Ausschüssen des Verwaltungs-/Aufsichtsrats verwiesen, 2005/162/EG, ABl. Nr. L 52, 51. Zur Diskussion, welchen Bestimmungen bei der Auslegung des Tatbestandsmerkmals im konkreten Fall Vorrang einzuräumen ist: HABERSACK, AG 2008, 98 ff; DERS., FS Goette, 2011, S. 121 ff (EUKommissionsempfehlung v. 15.2.2005); a. A. Bürgers/SchilHA, die für eine konzernrechtliche Auslegung der Unabhängigkeit plädieren und damit im Ergebnis den Empfehlungen in Ziff. 5.4.2. S. 2 den Vorzug geben, Bürgers/Schilha, AG 2010, 221, $229 \mathrm{f}$. Kritisch zu dieser doppelten Inbezugnahme des Gesetzgebers: JasPERs, AG 2009, 607, 610 f; Hennrichs, Stellungnahme zum RegE BilMoG v. 11.12. 2008, S. 13. abrufbar unter www.kapitalmarktrecht-im-internet.eu.

167 RegE BilMoG, S. $101 \mathrm{f}$.

168 RegE BilMoG, S. 102. 
Dies gilt unabhängig davon, ob diese Norm von der Entsprechungserklärung des $\$ 161$ AktG umfasst ist. Gleichzeitig macht er jedoch deutlich, dass sie im Einzelfall mit guter Begründung auch durchaus zu einer anderen Bewertung kommen können.

\section{c) Systematik}

Zudem können die Empfehlungen des DCGK eine Bindungswirkung entfalten, wenn sie ihrerseits Empfehlungen der EU-Kommission rezipieren. Wie gezeigt erzeugen die Empfehlungen der EU-Kommission Befassungs- und Befolgungspflichten ${ }^{169}$. Die Normenhierarchie verlangt daher eine systematische Berücksichtigung ${ }^{170}$. In der Vergangenheit hat die Regierungskommission Corporate Governance in verschiedenen Bereichen Anleihen bei Empfehlungen der EU-Kommission genommen und diese, zum Teil unverändert, als Empfehlung im DCGK übernommen ${ }^{171}$. So findet etwa die Empfehlung der Kommission zur Vergütungspolitik und Offenlegung von Vorstandsgehältern $^{172}$ ein Pendant im DCGK. Die in Ziff. 5.4.6 Abs. 3 enthaltene Empfehlung, die Vergütung von Aufsichtsratsmitgliedern individualisiert im Anhang oder im Lagebericht auszuweisen, stimmt inhaltlich mit Ziff. 5 der Empfehlung der Kommission überein, da mit „Mitgliedern der Unternehmensleitung " auch Aufsichtsratsmitglieder gemeint sind ${ }^{173}$. $\mathrm{Zu}$ nennen wäre auch die Unabhängigkeitsdefinition für Aufsichtsratsmitglieder in Ziff. 5.4.2 S. ${ }^{174}$, für die die Kommissionsempfehlung zur Unabhängigkeit von nicht-geschäftsführenden Direktoren und Aufsichtsratsmitgliedern Pate gestanden hatte ${ }^{175}$. Der DCGK bestimmt nun, dass ein Aufsichtsrat auch dann als nicht unabhängig einzustufen ist, wenn er in einer geschäftlichen oder persönlichen Beziehung zu einem kontrollierenden Aktionär oder einem mit diesem verbundenen Unternehmen steht, die einen wesentlichen und nicht nur vorüber-

169 S. oben II.1.c).

170 S. dazu auch: LeYens, aaO (Fn. 17), \$161 HGB Rdn. 114.

171 Wie eingehend die Auseinandersetzung mit den Empfehlungen der Kommission innerhalb der Regierungskommission erfolgt, lässt sich den Ausführungen von LUTTER, selbst ehemaliges Mitglied der Regierungskommission Corporate Governance, entnehmen: LutTER, EuZW 2009, 799, $804 \mathrm{f}$.

172 Empfehlung der Kommission zur Einführung einer angemessenen Regelung für die Vergütung von Mitgliedern der Unternehmensleitung börsennotierter Gesellschaften v. 14. 12. 2004, 2004/913/EG, ABl. Nr. L 385, 55.

173 Empfehlung 2004/913/EG v. 14. 12. 2004, ABl. Nr. L 385, 55, Ziff. 2.1; LutTEr, EuZW $2009,799,800$.

174 LutTER, EuZW 2009, 799, 804.

175 Empfehlung 2005/162/EG v. 15. 2. 2005, ABl. Nr. L 52, 51, Ziff. 13.1. iVm Anhang II. Vgl. dazu auch: Kremer, in: Ringleb/Cremer/Lutter/v. Werder, DCGK, 4. Aufl., 2010, Rdn. 1037. 
gehenden Interessenkonflikt begründen kann ${ }^{176}$. Mit Gegenargumenten lässt sich die Vermutungswirkung jedoch auch widerlegen, wie sogleich zu zeigen sein wird ${ }^{177}$.

\section{d) Telos}

Schließlich können auch teleologische Erwägungen zur Begründung einer eingeschränkten Bindungswirkung von Kodexempfehlungen herangezogen werden. Greift der Kodex Grundsätze auf, die in Literatur und Rechtsprechung allgemein anerkannt sind und damit best-practice darstellen ${ }^{178}$, so ist es gerechtfertigt, den jeweiligen Bestimmungen eine eingeschränkte Bindungswirkung als sekundäre Rechtsquelle zuzusprechen. So empfiehlt Ziff. 4.3.4 des DCGK, dass jedes Vorstandsmitglied Interessenkonflikte dem Aufsichtsrat gegenüber unverzüglich offen legt und die anderen Vorstandsmitglieder hierüber informiert. Die Kodexbestimmung greift dabei die organschaftliche Treuepflicht des Vorstandsmitglieds auf ${ }^{179}$ und hat damit als Seitenstück der organschaftlichen Sorgfaltspflicht Bedeutung für die Konkretisierung des generalklauselartigen Rechtsbegriffes in $\ 93$ Abs. 1 Satz 1 AktG. Diese Offenlegungspflicht entspricht der herrschenden Meinung im aktienrechtlichen Schrifttum ${ }^{180}$ und wurde auch bereits höchstrichterlich bestätigt ${ }^{181}$. In diesem Fall sprechen teleologische Gründe dafür, die Kodexempfehlung als sekundäre

176 Deutscher Corporate Governance Kodex in der Fassung vom 15. 5. 2012. Die Regierungskommission hat damit auf die im Rahmen des Konsultationsverfahrens vorgetragene Kritik reagiert und den Vorschlag, eine Gefährdung der Unabhängigkeit bereits bei 10-\% Beteiligung in der Regel anzunehmen, wieder verworfen, s. dazu noch Ziff. 5.4.2 S.2 Sp.3, Deutscher Corporate Governance Kodex in der Fassung vom 26.5. 2010 (mit Vorschlägen aus der Plenarsitzung vom 17.1.2012), abrufbar unter: http://www.corporate-governance-code.de/ger/download/aenderungen_2012/Kodexaenderungen_final_2012_02_01.pdf.

177 S. unten IV.4. Ähnlich Lieder, NZG 2005, 569, 570, der konstatiert, dass sich die „Interpretation von Ziff. 5.4.2 (...) zwar durchaus an europäischen Unabhängigkeitsstandards orientieren" könne, jedoch eine Abweichung von der Kommissionsempfehlung für gerechtfertigt hält, wenn, wie im dualistischen System, ein „vergleichbarer Unabhängigkeitsstandard bereits auf anderem Wege sichergestellt" sei.

178 Ähnlich Weber-Rey/Buckel, die Kodexempfehlungen eine mittelbare Haftungsrelevanz im Rahmen der business judgement rule des $₫ 93 \mathrm{AktG}$ zusprechen wollen, sofern sie best practice-Grundsätze darstellen, WeBER-Rey/Buckel, AG 2011, 845, $848 \mathrm{ff}$.

179 Nach h. M. wird die organschaftliche Treuepflicht durch den organschaftlichen Bestellungsakt begründet. Sie kann allerdings durch einzelne Regelungen des Anstellungsvertrages modifiziert werden. Fleischer, in: Spindler/Stilz, AktG, 2. Aufl., 2010, \93 Rdn. 117; WeLLER, ZHR 175 (2011), 110, 120.

180 Норт, ZGR 2004, 1, 31; Mertens/Cahn, Kölner Komm. z. AktG, 3. Aufl., 2010, \93 Rdn. 110; SpIndLER, in: Spindler/Stilz, AktG, 2. Aufl., 2010, \$93 Rdn. 124 („universal anerkanntes Verhaltensgebot").

181 BGHZ 20, 239, 246. 
Rechtsquelle einzustufen ${ }^{182}$. Entsprechende Bindungswirkung wird man somit auch den Parallelregelungen für Aufsichtsratsmitglieder in Ziff. 5.5.2 und 5.5.3 des DCGK attestieren können ${ }^{183}$.

\section{Feblende Bindungswirkung des DCGK}

\section{a) Bereich des extra legem}

Eine Einstufung als sekundäre Rechtsquelle wird man hingegen Kodexbestimmungen versagen müssen, die extra legem keinen Bezug zu gesetzlichen Regelungen aufweisen ${ }^{184}$. Auch der Regierungsauftrag an die Kodexkommission gibt vor, gesetzlich eröffnete Handlungsspielräume nur innerhalb des geltenden Rechts zu konkretisieren ${ }^{185}$. So sieht etwa $₫ 93$ Abs. 2 Satz 3 AktG lediglich für Vorstandsmitglieder einen obligatorischen Selbstbehalt in Höhe von $10 \%$ des von ihnen verursachten Schadens vor. Über diese gesetzliche Regelung hinaus empfiehlt Ziff. 3.8 Abs. 2 des DCGK auch für Aufsichtsratsmitglieder die Vereinbarung eines Selbstbehalts. Ein weiteres Beispiel: Nach der gesetzlichen Regelung des $\$ 90$ Abs. 1 Satz 3, Abs. 5 Satz 3 AktG hat der Vorstand dem Aufsichtsratsvorsitzenden über wichtige Anlässe, die die Gesellschaft oder ein verbundenes Unternehmen betreffen, zu berichten. Der Aufsichtsratsvorsitzende hat die übrigen Aufsichtsratsmitglieder spätestens in der nächsten [ordentlichen] Aufsichtsratssitzung zu unterrichten ${ }^{186}$. Nach der gesetzlichen Regelung wird dem Aufsichtsratsvorsitzenden also ein Ermessensspielraum eingeräumt, bis wann er die übrigen Mitglieder unterrichtet. In Ziff. 5.2 Abs. 2 des DCKG wird der Aufsichtsratsvorsitzende verpflichtet,

182 Indirekt bestätigt wird diese These auch durch die Ausführungen von Hopt, der den Kodexempfehlungen in Ziff. 4.3 eine Ausstrahlungswirkung auf $\$ \mathbb{S 7 6 , 9 3}$ und 116 AktG attestiert, soweit diese Empfehlungen nicht ohnehin schon dem aktienrechtlichen Schrifttum entsprechen würden. Es sei daher wenig wahrscheinlich, dass ein Gericht aktienrechtlich anders entscheiden würde, НоPT, ZGR 2004, 1, 22 f.

183 Ähnlich auch Hoffmann-Becking, der konstatiert, dass eine Pflicht zur Offenlegung von Interessenkonflikten, wie sie im Kodex für Aufsichtsratsmitglieder empfohlen wird, „ohnehin kraft Gesetzes“ bestehen würde, Hoffmann-Becking, Münchener Handbuch des Gesellschaftsrechts, Band 4, Aktiengesellschaft, 3. Aufl., 2008, 333 Rdn. 64.

184 S. auch die Selbsteinschätzung der Regierungskommission, die einen negativen Kriterienkatalog zur Unabhängigkeit von Aufsichtsratsmitgliedern in der jüngsten Neufassung mit dem Argument ablehnt, dass es dafür keine rechtliche Grundlage gebe, Presseinformation Regierungskommission Corporate Governance vom 16.5.2012, S.2, abrufbar unter: http://www.corporate-governance-code.de/ger/download/kodex_2012/Pressemitteilung_Kodexanpassungen_16_05_2012_de.pdf.

185 Ringleb, aaO (Fn. 113), Rdn. 17 u. 20.

186 Der Verweis in $\$ 90$ Abs. 5 Satz 3 AktG auf $\$ 90$ Abs. 1 Satz 2 AktG ist ein redaktionelles Versehen, vgl. Hüffer, aaO (Fn. 35), $\$ 90$ AktG Rdn. 14. 
„sodann“ den Aufsichtsrat zu unterrichten, was auf eine Pflicht zur unverzüglichen Informationsweiterleitung hinauslaufen würde ${ }^{187}$. Wenn der Kodex diesen Spielraum einschränkt, werden die Pflichten des Aufsichtsratsvorsitzenden verschärft ${ }^{188}$.

Eine Bindungswirkung für die Gerichte können sie aufgrund der problematischen verfassungsrechtlichen Legitimation nicht entfalten ${ }^{189}$.

\section{b) Bereich des contra legem}

Erst recht bindet eine contra legem Regelung nicht. Die Regierungskommission ist ebenso wie die Exekutive an das Grundgesetz und an parlamentarische Gesetze gebunden ${ }^{190}$. Nach $\ 33$ Abs. 1 Satz 2 WpÜG sind dem Vorstand der Zielgesellschaft einer Übernahme auch ohne Mitwirkung der Hauptversammlung und des Aufsichtsrates Handlungen eines ordentlichen und gewissenhaften Geschäftsleiters einer Gesellschaft gestattet. Die gesetzliche Regelung lässt damit auch außergewöhnliche Geschäfte, wie etwa einen Beteiligungserwerb, zu. Im Gegensatz dazu hieß es in Ziff. 3.7 Abs. 2 Satz 1 des DCGK, dass der Vorstand nach Bekanntgabe eines Übernahmeangebots keine Handlungen außerhalb des gewöhnlichen Geschäftsverkehrs vornehmen darf, durch die der Erfolg des Übernahmeangebots verhindert werden könnte, wenn er dazu nicht von der Hauptversammlung ermächtigt ist oder der Aufsichtsrat dem zugestimmt hat ${ }^{191}$. Die Bestimmung des DCGK stand damit im Widerspruch zur gesetzlichen Regelung ${ }^{192}$ und war daher als Normsetzung contra legem rechtswidrig. In der aktuellen Fassung vom 15. Mai 2012 wurde diese Bestimmung nunmehr korrigiert ${ }^{193}$. Durch den Vorbehalt anderweitiger gesetzlicher

187 Kritisch auch: Baums, Bericht der Regierungskommission Corporate Governance, 2001, Rdn. 29.

188 So auch: SEIBT, in: Hommelhoff/Hopt/v. Werder, Handbuch Corporate Governance, 2. Aufl., 2009, S. 406.

189 S. oben II.2.b); Zuletzt: Wernsmann/GatzKa, NZG 2011, 1001. S. dazu auch: HüFFER, aaO (Fn. 35), \161 AktG Rdn. 4 ff; Hoffmann-Becking, ZIP 2011, 1173, 1174; DERs., FS Hüffer, S. 337 f; Spindler, aaO (Fn. 20), $\$ 161$ AktG Rdn.11. a. A. HaberSACK, Gutachten DJT, 2012, E $53 \mathrm{f}$.

190 Hohl, aaO (Fn. 18), S. 77, der allerdings davon ausgeht, dass die Regierungskommission in einem bewusst vom Gesetzgeber überlassenen „gesetzesfreien Raum“ tätig ist.

191 So noch der Deutsche Corporate Governance Kodex i. d. F. v. 26. 5. 2010.

192 Hirte, Kölner Komm. z. WpÜG, 2. Aufl., 2010, \33 Rdn. 70; Hoffmann-Becking, ZIP 2011, 1173, 1175. In der Literatur werden noch folgende Bestimmungen des Kodex als unzutreffend und damit contra legem angesehen: Ziff. 2.1.2 S. 2, Ziff. 2.2.2., Ziff. 3.4., 3.9.; Ziff. 3.9., Ziff. 4.3.4., Ziff. 5.1.2. und Ziff. 5.5.4.; s. dazu SeIdeL, ZIP, 2004, 285, 292; KrIEgER, FS Ulmer, 2003, S. 365, 367.

193 Deutsche Corporate Governance Kodex in der Fassung vom 15. 5. 2012. 
Regelungen finden sich die Ausnahmen des $₫ 33$ Abs. 1 Satz 2 WpÜG nunmehr auch im Kodex wieder ${ }^{194}$.

\section{Zulässige Normkonkretisierung von gesetzlichen Generalklauseln durch private Regelsetzer}

Einfach gestaltet sich die Abgrenzung von intra und extra legem in den Fällen, in denen die privaten Regelwerke Normierungslücken schließen und die privaten Regulierungsgremien evident Normsetzung extra legem betreiben. Schwieriger sind jedoch die Fälle, in denen diese Anbindung an das Gesetz zwar besteht, die gesetzliche Regelung jedoch dermaßen unbestimmt ist, dass jeglicher Versuch der Konkretisierung einer eigenen Normerzeugung gleichkommt. In der juristischen Methodenlehre hat sich mittlerweile die Erkenntnis durchgesetzt, dass jeder Gesetzesauslegung und jeder Ausfüllung von unbestimmten Rechtsbegriffen und Generalklauseln ein rechtsschöpferisches Element des Rechtsanwenders innewohnt ${ }^{195}$. Vor allem bei Generalklauseln und Blankettnormen ${ }^{196}$ verliert die Dichotomie von Rechtserzeugung und Rechtsanwendung daher an Bedeutung. Die Normkonkretisierung ${ }^{197}$ erfolgt nicht deduktiv, vielmehr muss der Rechtsanwender dafür alle einschlägigen und sachlich aufschlussreichen Normen, Präjudizien oder andere Vergleichsfälle und Rechtsprinzipien heranziehen ${ }^{198}$. Deutlich wird diese Problematik bei der Frage, ob die Empfehlungen des DCGK zur Konkretisierung der allgemeinen Sorgfaltspflichten der $\$ \mathbb{S} 93,116$ AktG herangezogen werden sollen ${ }^{199}$. Die generalklauselartige Umschreibung der unternehmerischen Ver-

194 Ziff. 2 lit.f, Erläuterung zu den Änderungsvorschlägen der Plenarsitzung vom 17.2.2012, abrufbar unter: http://www.corporate-governance-code.de.

195 Engisch, Einführung in das juristisches Denken, 11. Aufl., 2010, S. 220; Rhinow, Rechtsetzung und Methodik, 1979, S. 177 ff; RöтнеL, aaO (Fn. 114), S. 20 ff.

196 Blankettnormen verweisen im Unterschied zu Generalklauseln auf eine Ergänzung im gleichen Gesetz oder in anderen Gesetzen und somit auf bereits positiviertes Recht. Generalklauseln verweisen hingegen auch auf außerrechtliche Sitten- und Verhaltensgebote. RGSt 46, 393, 395; RöTHeL, aaO (Fn. 114), S. 33 f.

197 Wenn der Wortlaut der Norm zu unbestimmt ist, spricht man nicht mehr von Auslegung, sondern von Konkretisierung des Rechts.

198 Bydlinsky, Juristische Methodenlehre und Rechtsbegriff, 2. Aufl., 1991, 582 f. Eine wichtige Rolle spielen in diesem Zusammenhang auch die Topik und die Fallvergleichsmethode.

199 Bejahend etwa LutTer, aaO (Fn. 27), Rdn. 1623; Ders., ZHR 166 (2002), 523, 540; Borges, ZGR 2003, 508, 521; SEIDEL, ZIP 2004, 285, 290; SEIBT, AG 2002, 249, 251; Schüppen, ZIP 2002, 1269, 1271; OLG Schleswig, NZG 2003, 176, 179; BGHZ 158, 122, 127; Kort, FS K. Schmidt, S. 945, 958 ff; Möllers, aaO (Fn. 12), S. 143, 147; a. A. HüFfer, aaO (Fn. 35), \$161 AktG Rdn. 4; BERG/STÖCKER, WM 2002, 1569, 1575 (nur wenn Handelsbräuche); Ulmer, ZHR 166 (2002), 150, 157; SpINDler, NZG 2011, $1007,1010$. 
haltenspflichten lädt geradezu dazu ein, einzelne Bestimmungen des DCGK ungeprüft und vorschnell zu deren Konkretisierung heranzuziehen.

Es bedarf daher weiterer Kriterien, anhand derer die Unterscheidung von Standardsetzung intra legem und extra legem vorgenommen werden kann, da ansonsten den privaten Gremien „Tür und Tor" für eigene gesetzgeberische Tätigkeit geöffnet werden würde. Darauf weist auch Lutter hin, wenn er in diesem Zusammenhang eine sorgfältige Einzelfallprüfung verlangt ${ }^{200}$, ob die Empfehlungen zur Ausfüllung dieser Generalklauseln geeignet sind und die allgemeinen Sorgfaltspflichten nicht bereits durch die Leitungsaufgabe des Vorstandes ( $\$ 76$ Abs. $1 \mathrm{AktG})$, seine Treuebindungen und gesetzliche Einzelvorschriften konkretisiert werden ${ }^{201}$.

Ausgangspunkt ist zunächst einmal das parlamentarische Gesetz selbst. Je höher dessen Regelungs- bzw. Programmierungsdichte ist und je detaillierter dessen Tatbestandsmerkmale sind, umso eher sind die sachlich einschlägigen privaten Bestimmungen dem Bereich der Normsetzung intra legem zuzuordnen $^{202}$. Stellt das Gesetz selbst einen solchen Rahmen nur unzureichend zur Verfügung, wie dies bei Generalklauseln und Blankettnormen zunächst einmal der Fall ist, so stellt sich die Frage, ob mit Hilfe von Präjudizien, herrschender Literaturansicht, allgemeinen Rechtsprinzipien oder internationalen Standards ein solcher Rahmen gefunden werden kann. Befindet sich nun der Regelungsgehalt der privaten Bestimmungen innerhalb dieses Rahmens, so ist sie dem Bereich der Normsetzung intra legem zuzuordnen. Diese Unterscheidung lässt sich an dem bereits vorgestellten Beispiel zur Offenlegung von Interessenkonflikten in Ziff. 4.3.4. abschließend verdeutlichen:

Ziff. 4.3.4 des DCGK empfiehlt, dass jedes Vorstandsmitglied Interessenkonflikte dem Aufsichtsrat gegenüber unverzüglich offen legt und die anderen Vorstandsmitglieder hierüber informiert.

Diese Offenlegungspflicht entspricht sowohl der h. M. im aktienrechtlichen Schrifttum ${ }^{203}$ als auch der höchstrichterlichen Rechtsprechung ${ }^{204}$, so dass sich mit Hilfe dieser beiden Faktoren ein Rahmen zur Ausfüllung der Generalklausel bestimmen lässt. Die Kodexempfehlung in Ziff. 4.3.4 ist daher dem Bereich der Normsetzung intra legem zuzuordnen. Greift der Kodex Grundsätze auf, die in Literatur und Rechtsprechung allgemein anerkannt sind und damit best-practice darstellen, so ist es gerechtfertigt, den jeweiligen Bestim-

200 Lutter, aaO (Fn. 27), Rdn. 1624; Doralt, aaO (Fn. 27), \$13 Rdn. 181.

201 Hüffer, aaO (Fn. 35), $\$ 93$ AktG Rdn. 3 a.

202 S. bereits oben IV.2.a).

203 Fleischer, WM 2003, 1045, 1050; Hopt, ZGR 2004, 1, 31; Mertens/Cahn, aaO (Fn. 180), $\$ 93$ Rdn. 110.

204 BGHZ 20, 239, 246. 
mungen eine eingeschränkte Bindungswirkung als sekundäre Rechtsquelle zuzusprechen.

\section{Widerlegung der Vermutungswirkung}

\section{a) Durch eine überzengende Gegenposition}

Die Einstufung einer Kodexempfehlung als sekundäre Rechtsquelle bedeutet nicht, dass die Gerichte gezwungen wären ihr sklavisch zu folgen. Vielmehr besteht die Möglichkeit, die einzelne Empfehlung der Regierungskommission mit guten Argumenten zu widerlegen.

So haben die Gerichte zwar auch die erst vor kurzem eingeführte Empfehlung in Ziff. 5.4.2. Satz 2 zu berücksichtigen, wonach Aufsichtsräte insbesondere dann nicht als unabhängig anzusehen seien, wenn sie in einer persönlichen oder geschäftlichen Beziehung zu einem kontrollierenden Aktionär stehen ${ }^{205}$. Dennoch bedeutet auch eine solche Bestimmung nicht, dass die Gerichte sie ihrer Urteilsfindung, etwa im Rahmen von Anfechtungsklagen gegen die Wahl eines unabhängigen Finanzexperten nach $\$ 100 \mathrm{Abs.} 5 \mathrm{AktG}$, automatisch zugrunde legen müssten ${ }^{206}$. Vielmehr kann diese Empfehlung der Regierungskommission mit besseren Gründen widerlegt werden. Damit obliegt es vor allem der Partei, zu deren Ungunsten die Vermutung wirkt, das Gericht in dessen Entscheidungsfindung zu beeinflussen. Als Gegenargumente kommen insbesondere telelogische Erwägungen in Betracht, wie der Umstand, dass der Minderheitenschutz bereits durch das deutsche Aktien- und Konzernrecht in ausreichendem Maße berücksichtigt wird ${ }^{207}$. Mögliche Konflikte zwischen Gesellschaft und Großaktionär werden im Aktiengesetz in den Vorschriften des Konzernrechts geregelt ${ }^{208}$. Ein Abstellen auf die Besonderheiten des deutschen Konzernrechts hätte daher, in Anbetracht der dogmatischen Bedenken, die mit einer Kodexempfehlung besagten Inhalts verbunden sind, durchaus gute Chancen, das Gericht von der „Unrichtigkeit“ der Empfehlung zu überzeugen.

Aus rechtspolitischer Sicht wäre es daher wünschenswert, wenn die Regierungskommission in Zukunft ihre Kodexbestimmungen mit einem Begrün-

205 S. oben IV.2.c).

206 Zur Problematik der Anfechtbarkeit von Hauptversammlungsbeschlüssen bei der Wahl des unabhängigen Finanzexperten i. S.d. $\$ 100$ Abs. 5 AktG: Gesell, ZGR 2011, $361,391 \mathrm{ff}$.

207 Hoffmann-Becking, ZGR 2004, 355, 359 f; HüfFer, ZIP 2006, 637, 642; Bürgers/ Schilha, AG 2010, 221, 229 f; KrIEger, ZGR 2012, 202, 211; s. zur Diskussion auch: LutTer, EuZW 2009, 799 ff; DERs., EuZW 2010, 87 ff; Wackerbarth, EuZW 2010, $46 \mathrm{ff}$.

208 Stellungnahme DAV Handelsrechtsausschuss v. 1.2. 2012, NZG 2012, 335, 338. 
dungsteil versehen würde. Dieser würde es den Normbetroffenen erleichtern, sich mit den Empfehlungen argumentativ auseinander zu setzen um deren Vermutungswirkung zu widerlegen. Eine solche Begründungspflicht findet sich auch auf europäischer Ebene: Gem. Art. 296 Abs. 2 AEUV sind Unionsorgane verpflichtet, ihre Rechtsakte mit Begründungen zu versehen, um den Gedankengang des Gesetzgebers oder der Behörde nachzuvollziehen ${ }^{209}$.

\section{b) Durch eine abweichende Begründung in der Entsprechenserklärung}

$\mathrm{Zu}$ thematisieren ist schließlich, wie sich das „Explain“ des $\$ 161 \mathrm{AktG}$ auswirkt, wenn also das Unternehmen erklärt, von den Vorgaben des DCGK abweichen zu wollen. Zum Teil wird vertreten, das bewusst erklärte Abweichen von den Vorgaben des DCGK wäre haftungsrechtlich unbeachtlich, weil der DCGK rechtlich irrelevant $\operatorname{sei}^{210}$. Wegen des Abweichens könne dem Vorstand bzw. dem Aufsichtsrat des Unternehmens kein Sorgfaltspflichtverstoß angelastet werden, weil das Abweichen ja gerade begründet wurde ${ }^{211}$.

Nach der Gegenansicht kann die erklärte Nichtbeachtung einer Kodexempfehlung durchaus den Vorwurf sorgfaltswidrigen Verhaltens von Vorstand und Aufsichtsrat begründen ${ }^{212}$. Dies gelte vor allen dann, wenn die Kodexempfehlungen Grundsätze ordnungsgemäßer Unternehmensführung widerspiegeln oder konkretisieren würden ${ }^{213}$.

Diese Ansicht verdient Zustimmung ${ }^{214}$. Sekundäre Rechtsquellen begründen für die Gerichte eine Befassungs- und subsidiäre Befolgungspflicht, unabhängig von einer zuvor erklärten Abweichung durch das Unternehmen ${ }^{215}$. Dies bedeutet, dass das Gericht sich mit den Empfehlungen des Kodex zu befassen und argumentativ auseinanderzusetzen hat ${ }^{216}$, die Partei, zu deren Ungunsten

209 S. dazu: Calliess, in: Calliess/Ruffert, EUV AEUV, 4. Aufl., 2011, Art. 296 AEUV Rdn. 15. Während ex-Art. 253 EGV hiervon auch Empfehlungen und Stellungnahme umfasste, soll der nun verwendete Begriff der „Rechtsakte“ diese nicht mehr umfassen, s. etwa Gellermann, in: Streinz, EUV/AEUV, 2. Aufl., 2012, Art. 296 Rdn. 4.

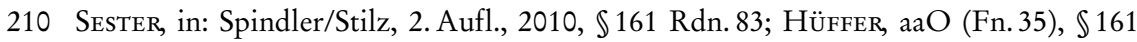
AktG Rdn. $26 \mathrm{f}$.

211 Spindler, aaO (Fn. 20), $\$ 161$ AktG Rdn.68; Hüffer, aaO (Fn.35), $\$ 161$ AktG Rdn. 27; EtTINGER/GrütZediek, AG 2003, 353, $354 \mathrm{f}$.

212 KORT, FS K. Schmidt, S. 945, 949 u. 959; LUTTER, ZHR 166 (2002), 523, 542; SCHÜPPEN ZIP 2002, 1269, 1271.

213 Kort, FS K. Schmidt, S. 945, 961.

214 A. A. Sester, aaO (Fn. 210), $\$ 161$ AktG Rdn. 83; Hüffer, aaO (Fn. 35), \$161 AktG Rdn. 26, die jedoch beide, von der rechtlichen Unverbindlichkeit der Kodexempfehlungen ausgehend, zu dem Ergebnis kommen, dass eine erklärte Nichtbeachtung nicht haftungsrelevant sein kann.

215 S. oben II.3.

216 S. oben II.3.a). 
die Vermutung wirkt, hingegen im Rechtstreit die Möglichkeit bekommt, die Vermutungswirkung mit überzeugender Gegenargumentation zu widerlegen $^{217}$. Für eine solche Sichtweise streitet auch die Überlegung, dass nicht die Gerichte sondern die gegenwärtigen und zukünftigen Aktionäre des Unternehmens Hauptadressaten der Entsprechenserklärung sind ${ }^{218}$.

\section{Zusammenfassung und Ausblick}

\section{Zur Argumentationslast}

Mit der klassischen Dichotomie von hard law und soft law, Rechtsquellen und Rechtserkenntnisquellen, lässt sich das Phänomen privater Normsetzung nicht mehr erfassen. Die Lehre von den sekundären Rechtsquellen geht über die klassische Dichotomie hinaus und begründet eine dritte Kategorie von Recht, die mehr ist als soft law, aber weniger als eine verbindliche Rechtsquelle ${ }^{219}$. Die Gerichte trifft in diesem Falle, ähnlich wie im Bereich des Richterrechts, eine Befassungspflicht sowie eine subsidiäre Befolgungspflicht. Will der Adressat der Empfehlung nicht folgen, muss er dies gegenüber dem Gericht begründen. Damit unterscheidet sich die private Rechtsetzung deutlich von Rechtserkenntnisquellen, wie etwa ausländische Urteile oder die Rechtsliteratur, mit denen sich der Rechtsanwender argumentativ auseinandersetzen kann, aber nicht muss.

\section{Intra legem-Anbindung von Standards an das Gesetz}

Für die einzelnen Bestimmungen dieser privaten Regelwerke lässt sich zwischen intra legem, extra legem und contra legem unterscheiden. Im Bereich der Normsetzung intra legem werden einzelne Rechtsnormen ausgelegt sowie unbestimmte Rechtsbegriffe und Generalklauseln konkretisiert. Die Normsetzung erfolgt damit innerhalb des tatbestandlichen Anwendungsbereiches eines Gesetzes ${ }^{220}$. Im Bereich der Normsetzung extra legem werden hingegen

217 S. oben II.3.b). Das Unternehmen kann die aus seiner Sicht wesentlichen Gegenargumente auch bereits in der Entsprechenserklärung vortragen. Insofern ist der Vorschlag der Europäischen Kommission, im Rahmen der Entsprechenserklärung nicht nur die Abweichung, sondern auch die eigene Lösungsalternative zu begründen, ausdrücklich zu begrüßen, Europäische Kommission, Grünbuch Europäischer Governance-Rahmen, KOM 2011, S. 22; S. dazu: Habersack, Gutachten DJT 2012, E 49 u. E 57.

218 Auch durch die im Zuge des BilMoG eingeführte Begründungspflicht in $\ 161$ Abs. 1 S. 1 AktG hat sich an der Informationsfunktion der Entsprechenserklärung nichts geändert, s. Begr. RegE BilMoG, BT-Drs. 16/10067, S. 103 f.

219 Möllers, FS Buchner, S. 649, 654 ff; DERs., aaO (Fn. 12), S. 143, 150 ff.

220 S. II.2.b)aa). 
neue Verhaltensanforderungen an die Adressaten der Regelwerke gestellt, die über die bestehende Gesetzeslage hinausgehen ${ }^{221}$. Bestimmungen, die der geltenden Rechtslage widersprechen und daher contra legem erlassen wurden, sind schließlich generell unzulässig.

Will der Gesetzgeber die private Normsetzung rezipieren, kann er die Normen des DCKG unmittelbar verrechtlichen, wie dies bereits in der Vergangenheit durch das VorstOG ${ }^{222}$ und das VorstAG ${ }^{223}$ geschah. Daneben wäre es auch möglich, eine neue Generalklausel zu schaffen, auf die der DCGK dann Bezug nehmen kann ${ }^{224}$.

Die „wesentlichen“ Entscheidungen muss der Gesetzgeber auch weiterhin durch Gesetz treffen. Aus diesem Grunde kann lediglich der privaten Normsetzung intra legem eine mittelbar rechtliche Bindungswirkung als sekundäre Rechtsquelle zugestanden werden, da in diesem Falle eine Rückbindung an das demokratisch legitimierte Gesetz besteht. Private Normsetzung extra legem kann von den Gerichten lediglich als Rechtserkenntnisquelle herangezogen werden. Private Normsetzung, die gegen gesetzliche Bestimmungen verstößt (contra legem) und damit rechtswidrig ist, darf von den Gerichten überhaupt nicht herangezogen werden. Mit diesen Überlegungen wird auch den verfassungsrechtlichen Bedenken privater Rechtsetzung Rechnung getragen.

221 S. II.2.b)bb).

222 Gesetz über die Offenlegung der Vorstandsvergütungen (Vorstandsvergütungs-Offenlegungsgesetz - VorstOG) v. 3. 8.2005, BGBI. I 2267.

223 Gesetz zur Angemessenheit der Vorstandsvergütung (VorstAG) v. 31. 7. 2009, BGB1. I 2509.

224 Eine Kodexbestimmung, die für die Besetzung von Vorständen und Aufsichtsräten börsennotierter Unternehmen eine bestimmte Frauenquote empfiehlt, wäre etwa gesetzesübersteigend (extra legem), wenn der Gesetzgeber eine solche Bestimmung im Aktienrecht nicht bereits in irgendeiner Weise normiert hätte. Denkbar wäre jedoch, dass der Gesetzgeber in den $\int \mathbb{S} 84$ Abs. 1 und 96 AktG eine „angemessene Berücksichtigung" der Geschlechter bei der Besetzung von Vorständen und Aufsichtsräten vorschreiben würde. Enthielte nun der DCGK eine Empfehlung dahingehend, bei der Besetzung dieser beiden Gremien eine starre Frauenquote von $40 \%$ einzuhalten, so würde es sich dabei um Normsetzung intra legem handeln. In seiner aktuellen Fassung beschränkt sich der Kodex in Ziff. 4.1.5., 5.1.2. und 5.4.1 auf die Empfehlungen, bei der Besetzung von Führungsfunktionen im Unternehmen, Vorstand und Aufsichtsrat auf Vielfalt (Diversity) zu achten und dabei insbesondere eine angemessene Berücksichtigung von Frauen anzustreben und vermeidet daher eine starre Frauenquote. S. zur aktuellen Diskussion Möllers/Hailer, JZ 2012, 841, $847 \mathrm{f}$. 